math.QA/0412113

\title{
GLOBAL GEOMETRIC DEFORMATIONS OF CURRENT ALGEBRAS AS KRICHEVER-NOVIKOV TYPE ALGEBRAS
}

\author{
ALICE FIALOWSKI AND MARTIN SCHLICHENMAIER
}

\begin{abstract}
We construct algebraic-geometric families of genus one (i.e. elliptic) current and affine Lie algebras of Krichever-Novikov type. These families deform the classical current, respectively affine Kac-Moody Lie algebras. The construction is induced by the geometric process of degenerating the elliptic curve to singular cubics. If the finitedimensional Lie algebra defining the infinite dimensional current algebra is simple then, even if restricted to local families, the constructed families are non-equivalent to the trivial family. In particular, we show that the current algebra is geometrically not rigid, despite its formal rigidity. This shows that in the infinite-dimensional Lie algebra case the relations between geometric deformations, formal deformations and Lie algebra twocohomology are not that close as in the finite-dimensional case. The constructed families are e.g. of relevance in the global operator approach to the Wess-Zumino-Witten-Novikov models appearing in the quantization of Conformal Field Theory.
\end{abstract}

\section{INTRODUCTION}

Deformations of mathematical structures is a tool of fundamental importance in most parts of mathematics, in mathematical physics, and physics. Via deforming the object into a "similar" object, we get a richer picture about the original object itself. Moreover, via deformations we can approach the problem whether we can equip the set of mathematical structures under consideration (maybe up to certain equivalences) with the structure of a topological or even geometric space. In other words, does there exist a moduli space for these structures? If so, then for a fixed object the deformations of this object should reflect the local structure of the moduli space at the point corresponding to this object.

This aspect of the theory of deformations originated with the problem of classifying all possible pairwise non-isomorphic complex structures on a given differentiable real manifold. The fundamental idea, which should be credited to Riemann, was to introduce an analytic structure therein.

Date: 2.12 .2004

1991 Mathematics Subject Classification. Primary: 17B66; Secondary: 17B56, 17B65, 17B68, 14D15, 14H52, 30F30, 81T40 .

Key words and phrases. Deformations of algebras; rigidity; affine Lie algebra; Kac-Moody algebras; current algebras; Wess-Zumino-Witten-Novikov models; Krichever-Novikov algebras; elliptic curves; Lie algebra cohomology; conformal field theory. 
The notion of local and infinitesimal deformations of a complex analytic manifold first appeared in the work of Kodaira and Spencer. In particular, they proved that infinitesimal deformations can be parameterized by a corresponding cohomology group. The deformation theory of compact complex manifolds was developed further by Kuranishi and Palamodov. Shortly after the work of Kodaira and Spencer, algebro-geometric foundations were systematically developed by Grothendieck. For algebraic manifolds, the theory was constructed by Artin and Schlessinger.

A typical example for results in these directions is the following. For the moduli space $\mathcal{M}_{g}$ of smooth projective curves of genus $g$ over $\mathbb{C}$ (or equivalently, compact Riemann surfaces of genus $g$ ) the tangent space $T_{[C]} \mathcal{M}_{g}$ can be naturally identified with $\mathrm{H}^{1}\left(C, T_{C}\right)$, where $T_{C}$ is the sheaf of holomorphic vector fields over $C$. This extends to higher dimension. In particular, it turns out that for compact complex manifolds $M$, the condition $\mathrm{H}^{1}\left(M, T_{M}\right)=\{0\}$ implies that $M$ is rigid, [19, Thm. 4.4]. Rigidity means that any differentiable family $\pi: \mathcal{M} \rightarrow B \subseteq \mathbb{R}, 0 \in B$ which contains $M$ as the special member $M_{0}:=\pi^{-1}(0)$ is trivial in a neighbourhood of 0 , i.e. for $t$ small enough $M_{t}:=\pi^{-1}(t) \cong M$. Even more generally, for a compact complex manifold $M$ and $\mathrm{H}^{1}\left(M, T_{M}\right) \neq\{0\}$ there exists a versal family which can be realized locally as a family over a certain subspace of $\mathrm{H}^{1}\left(M, T_{M}\right)$ such that every appearing deformation family is "contained" in this versal family (see also [23] for definitions, results, and further references).

In this article we deal with deformations of algebraic structures, more specifically with deformations of Lie algebras, in particular with such of infinite dimension. Formal deformations of arbitrary rings and associative algebras, and the related cohomology questions, were first investigated by Gerstenhaber, in a series of articles [12. The notion of deformation was applied to Lie algebras by Nijenhuis and Richardson [21], 22].

The cohomology space related to the deformations of a Lie algebra $\mathcal{L}$ is the Lie algebra two-cohomology $\mathrm{H}^{2}(\mathcal{L}, \mathcal{L})$ of $\mathcal{L}$ with values in the adjoint module. Indeed, as long as this space is finite-dimensional, it gives all infinitesimal deformations (i.e. there exists a universal family of infinitesimal deformations). Even more, in this case there exists a versal family for the formal deformations with base in this cohomology space, see results of Fialowski [6, Fialowski and Fuchs 9], from where Theorem 2.15] below is quoted.

The positive results in the deformation theory of compact analytic manifolds lead to the impression that the vanishing of the relevant cohomology spaces will imply rigidity with respect to deformations also in the case of other structures. In an earlier paper [10] the authors showed that this hope is too naive. There we considered the (infinite dimensional) Witt algebra (respectively its universal central extension, the Virasoro algebra) and constructed algebraic-geometric deformations of it (each of them parameterized by the points of the affine line) using Krichever-Novikov vector field algebras. These deformations are non-trivial, only the special element in these families will be isomorphic to the Witt algebra, despite the fact that for the Witt algebra the two-cohomology space vanishes [7. Hence, in the case of infinite dimensional Lie algebras the vanishing of the two-cohomology only implies infinitesimal and formal rigidity. Formal rigidity means that every deformation over the algebra of formal power series in finitely many variables is equivalent to a trivial family (see Section 2 for precise definitions). Our example shows that the Witt (or Virasoro) algebra despite its formal rigidity is geometrically not rigid. 
This situation is peculiar for the case of infinite-dimensional Lie algebras. For finitedimensional Lie algebras we have strong relations between the different concepts of rigidity. In particular, if a finite-dimensional Lie algebra is non-singular (see Section 2 for the definitions and further details) it is infinitesimally, formally, geometrically, and analytically rigid if and only if the two-cohomology space vanishes.

In this article we elaborate further on these phenomena. We will consider the case of current algebras $\overline{\mathfrak{g}}=\mathfrak{g} \otimes \mathbb{C}\left[z^{-1}, z\right]$ and their central extensions $\widehat{\mathfrak{g}}$, the affine Lie algebras. Here $\mathfrak{g}$ is a finite-dimensional Lie algebra. Given an invariant, symmetric bilinear form $\beta$ the central extension $\widehat{\mathfrak{g}}$ is the vector space $\overline{\mathfrak{g}} \oplus t \mathbb{C}$ endowed with the Lie bracket

$$
\left[x \otimes z^{n}, y \otimes z^{m}\right]=[x, y] \otimes z^{n+m}-\beta(x, y) \cdot n \cdot \delta_{m}^{-n} \cdot t, \quad[t, \widehat{\mathfrak{g}}]=0, \quad x, y \in \mathfrak{g}, n, m \in \mathbb{Z} .
$$

These algebras are of fundamental importance in a number of fields. They supply examples of infinite dimensional algebras which are still accessible to a structure theory. They appear as gauge algebras in Conformal Field Theory (CFT), 1]. More generally, they are symmetry algebras of infinite-dimensional systems, integrable systems, etc. More examples can be found in the book of Kac [16. For simple finite-dimensional Lie algebras $\mathfrak{g}$ the central extensions $\widehat{\mathfrak{g}}$, the associated affine Lie algebras, are the Kac-Moody algebras of untwisted affine type.

For $\mathfrak{g}$ finite-dimensional and simple (and hence rigid) it was shown by Lecomte and Roger [20] that the current algebra $\overline{\mathfrak{g}}$ remains formally rigid. But we will exhibit again natural algebraic families of algebras containing $\overline{\mathfrak{g}}$ as special element but all other members will not be isomorphic to $\overline{\mathfrak{g}}$. In particular, these families can not be algebraic-geometrically equivalent to the trivial family. Hence, the current algebra $\overline{\mathfrak{g}}$ will be geometrically not rigid. All these families can be extended to families of centrally extended algebras and yield in this way nontrivial deformations of the affine algebra $\widehat{\mathfrak{g}}$. The families constructed appear as families of higher-genus multi-point current algebras of Krichever-Novikov type, see Section 3 for their definition. Hence, they are not just abstract families, but families obtained by geometric process. The results obtained do not have only relevance to the deformation theory of algebras, but are of importance in the fields in which current and affine algebras play a role. Note that the maximal nilpotent Lie algebra of $\overline{\mathfrak{g}}$ is even not formally rigid anymore, and its formal deformations are described in [4].

In particular, they are of relevance in two-dimensional CFT and its quantization. It is well-known that the Witt algebra, the Virasoro algebra, and their representations are of fundamental importance for conformal field theory on the Riemann sphere (i.e. for genus zero), see [1. Krichever and Novikov [18] proposed in case of higher genus Riemann surfaces (with two insertion points) the use of global operator fields which are given with the help of the Lie algebra of vector fields of Krichever-Novikov type, certain related algebras, and their representations (Section 3).

Their approach was extended to the multi-point situation (i.e. an arbitrary number of insertion points was allowed) by Schlichenmaier [27, 28], 29], 30. The necessary central extensions where constructed and higher genus multi-point current and affine algebras were introduced 32]. These algebras consist of meromorphic objects on a Riemann surface which are holomorphic outside a finite set $A$ of points. In turn the set $A$ is divided into two disjoint subsets $I$ and $O$. With respect to some possible interpretation of the Riemann surface as the world-sheet of a string, the points in $I$ are called in-points, the points in 
$O$ are called out-points, corresponding to incoming and outgoing free strings; the worldsheet itself corresponds to possible interaction. This splitting introduces an almost-graded structure (see Section 31) for the algebras and their representations. Such an almost-graded structure is needed to construct representations of relevance in the context of CFT, e.g. highest weight representations, fermionic Fock space representations, etc.

In the process of quantization of conformal fields one has to consider families of algebras and representations over the moduli space of compact Riemann surfaces (or equivalently, of smooth projective curves over $\mathbb{C}$ ) of genus $g$ with $N$ marked points. Models of most importance in CFT are the Wess-Zumino-Witten-Novikov models (WZWN). Tsuchiya, Ueno, and Yamada 38 gave a sheaf version of WZWN models over the moduli space. In [36], 37] Schlichenmaier and Sheinman developed a global operator version. In this context of particular interest is the situation $I=\left\{P_{1}, \ldots, P_{K}\right\}$, the marked points we want to vary, and $O=\left\{P_{\infty}\right\}$, a reference point. We obtain families of algebras over the moduli space $\mathcal{M}_{g, K+1}$ of curves of genus $g$ with $K+1$ marked points, and we are exactly in the middle of the main subject of this article. In [36 and 37 it is shown that there exists a global operator description of WZWN models with the help of the Krichever Novikov objects at least over a dense open subset of the moduli space. The following is just a very rough outline. Let us start from families of representations $\mathcal{V}$ of families of higher genus affine algebras (see Section 3 for their definition). The vector bundle of conformal blocks can be defined as the vector bundle with fiber (over the moduli point $\left.b=\left[\left(M,\left\{P_{1}, \ldots, P_{K}\right\},\left\{P_{\infty}\right\}\right)\right]\right)$ as follows. Take the quotient of the fiber of the representation $\mathcal{V}_{b}$ by the subspace generated by the vectors obtained by the action of those elements of the affine algebra which vanish at the reference point $P_{\infty}$ (i.e. the fiber of the quotient is the space of coinvariants of this subalgebra).

The bundle of conformal blocks carries a connection called the Knizhnik-Zamolodchikov connection. In its definition the Sugawara construction plays an important role, which associates to representations of affine algebras representations of the (almost-graded) centrally extended vector field algebras, see [35]. A certain subspace of the vector field algebra (assigned to the moduli point $b$ ) corresponds to tangent directions on the moduli space $\mathcal{M}_{g, K+1}$ at the point $b$.

Now clearly, the following question is fundamental. What happens if we approach the boundary of the moduli space? The boundary components correspond to curves with singularities. Resolving the singularities yields curves of lower genera. By geometric degeneration we obtain families of (Lie) algebras containing a lower genus algebra (or sometimes a subalgebra of it), corresponding to a suitable collection of marked points, as special element. Or reverting the perspective, we obtain a typical situation of the deformation of an algebra corresponding in some way to a lower genus situation, containing higher genus algebras as the other elements in the family. Such kind of geometric degenerations are fundamental if one wants to prove Verlinde type formula via factorization and normalization technique, see 38.

By a maximal degeneration a collection of $\mathbb{P}^{1}(\mathbb{C})$ 's will appear. Indeed, the examples considered in this article are exactly of this type. The deformations appear as families of current algebras which are naturally defined over the moduli space of genus one curves (i.e. of elliptic curves, or equivalently of complex one-dimensional tori) with two marked points. These deformations are associated to geometric degenerations of elliptic curves to 
singular cubic curves. The desingularization (or normalization) of their singularities will yield the projective line as normalization. We will end up with algebras related to the genus zero case. The full geometric picture behind the degeneration was discussed in 31 . In particular, we want to point out, that even if one starts with two marked points, by going to the boundary of the moduli space one is forced to consider more points (now for a curve of lower genus).

In special cases the classical current (or affine) algebras appear as degenerations of elliptic two-point current algebras. Considered from the opposite point of view, in the sense of this article, the elliptic two-point current algebras are global deformations of the classical current algebra. Nevertheless, as we show here, the structure of these algebras are not determined by the classical current algebras, despite the formal rigidity of the latter (if $\mathfrak{g}$ is simple), 20].

The structure of the article is the following. In Section 2 we recall the different definitions of deformations of Lie algebras. In particular we stress the fact, that in the case of infinite dimensional Lie algebra it is important to distinguish clearly the different notions, and indicate in which category we work: infinitesimal, formal, geometric, or analytic deformations. Also it is important to allow as base of the deformations not only local algebras, but also global algebras. They correspond to geometric situations. The most simple global case is the case of the algebra of polynomials in one variable. Geometrically this corresponds to a deformation over the affine line. As already indicated above, in the formal case everything can be described in cohomological terms. To contrast the infinite dimensional case with the finite-dimensional one, we recall some results from their theory. In the finite-dimensional case for non-singular Lie algebras all notions of rigidity introduced above are equivalent and correspond to the fact that the cohomology space vanishes. The reason is that in this case the whole situation can be described within the frame of finite-dimensional algebraic geometry.

In Section 3 we recall what is needed about the higher genus multi-point algebras of Krichever-Novikov type. The following algebras are introduced: the associative algebra of functions and the Lie algebras of vector fields and of currents, including their central extensions. For the currents the central extensions are the higher genus multi-point affine algebras.

In Section 4 we construct geometric deformations of the standard current algebra by considering certain families of algebras for the genus one case (i.e. the elliptic curve case) and let the elliptic curve degenerate to a singular cubic. The two points, where poles are allowed, are the zero element of the elliptic curve (with respect to its group structure) and a 2-torsion point. In this way we obtain families parameterized over the affine line with the peculiar behaviour that every family is a global deformation of the classical current algebra, i.e. the classical current algebra is a special member, whereas all other members are mutually isomorphic but not isomorphic to the special element if the finite-dimensional Lie algebra is simple, see Theorem 4.8. Even if restricted to small open neighbourhoods of the point corresponding to the special element, these families are non-trivial, only infinitesimally and formally they are trivial. The construction can be extended to the centrally extended algebras, yielding global deformations of the affine algebra. 
In Section [5] we consider the geometric picture behind it. In particular, we identify those algebras we obtain over the nodal cubics (i.e. the cubic curves with one singular point with two tangent directions at this point). We explain the geometric reason why we obtain them. Depending whether the node will become the point where a pole is allowed or not, we obtain a three-point current algebra of genus zero or a certain subalgebra of the classical current algebra.

In Section [6 we give the cohomology classes of our families of deformations. As it is known that these algebras are formally rigid in the simple case, it can be expected that, in general, the cocycles will be coboundaries. We show this by direct calculations.

In an appendix we calculate the cocycle defining the central extensions of our family.

In the process of constructing our families we constructed families of the commutative and associative algebra of functions on Riemann surfaces with prescribed regularity. These are families deforming the algebra of Laurent polynomials $\mathbb{C}\left[z^{-1}, z\right]$. This algebra is the coordinate algebra of the smooth affine curve $\mathbb{P}^{1} \backslash\{0, \infty\}$. The cohomology corresponding to commutative and associative deformations (i.e. the Harrison cohomology) vanishes for such algebras. The algebra is infinitesimally and formally rigid. Nevertheless, we constructed nontrivial (geometrically) local families. Maybe, Kontsevich's concept of semiformal deformations [17] (related to filtrations of certain type) might help to describe this situation better. Indeed, by the almost-grading of the families considered in this article, the families of associative algebras are semi-formal deformations in his sense.

Acknowledgements: The authors thank different institutions for hospitality experienced during the preparation of this article. A. F. and M. Sch. thank the Erwin-Schrödinger Institute (ESI) in Vienna, M. Schl. the Eötvös Loránd University in Budapest, and the Institut des Hautes Études Scientifiques (IHES) in Bures-sur-Yvette. Discussions with M. Kontsevich, P. Michor and E. Vinberg are gratefully acknowledged. The work was partially supported by grants OTKA T034641 and T043034.

\section{Some Generalities on Deformations of LiE Algebras}

\subsection{Intuitive description.}

Let us start with the intuitive definition of a Lie structure depending on a parameter t. Let $\mathcal{L}$ be a Lie algebra with Lie bracket $\mu=\mu_{0}$ over a field $\mathbb{K}$. A deformation of $\mathcal{L}$ is a one-parameter family $\mathcal{L}_{t}$ of Lie algebras (with the same underlying vector space) with the bracket

$$
\mu_{t}=\mu_{0}+t \phi_{1}+t^{2} \phi_{2}+\ldots
$$

where $\phi_{i}$ are $\mathcal{L}$-valued (alternating) two-cochains, i.e. elements of $\operatorname{Hom}_{\mathbb{K}}\left(\bigwedge^{2} \mathcal{L}, \mathcal{L}\right)=$ $C^{2}(\mathcal{L}, \mathcal{L})$, and $\mathcal{L}_{t}$ is a Lie algebra for each $t \in \mathbb{K}$. (see [5] 12]). In particular, we have $\mathcal{L}=\mathcal{L}_{0}$. Two deformations $\mathcal{L}_{t}$ and $\mathcal{L}_{t}^{\prime}$ are equivalent if there exists a linear automorphism $\hat{\psi}_{t}=\mathrm{id}+\psi_{1} t+\psi_{2} t^{2}+\ldots$ of $\mathcal{L}$ where $\psi_{i}$ are linear maps over $\mathbb{K}$, i.e. elements of $C^{1}(\mathcal{L}, \mathcal{L})$, such that

$$
\mu_{t}^{\prime}(x, y)=\hat{\psi}_{t}^{-1}\left(\mu_{t}\left(\hat{\psi}_{t}(x), \hat{\psi}_{t}(y)\right)\right)
$$

These objects are related to Lie algebra cohomology. We recall the following definitions. A bilinear map $\omega: \mathcal{L} \otimes \mathcal{L} \rightarrow \mathcal{L}$ is a Lie algebra two-cocycle with values in the adjoint 
representation $\mathcal{L}$, if $\omega$ is alternating and fulfills

$$
\begin{aligned}
0=d_{2}(\omega)(x, y, z):=\omega([x, y], z)-\omega & ([x, z], y)+\omega([y, z], x) \\
& -[x, \omega(y, z)]+[y, \omega(x, z)]-[z, \omega(x, y)] .
\end{aligned}
$$

The vector space of two-cocycles is denoted by $\mathrm{Z}^{2}(\mathcal{L}, \mathcal{L})$. A two-cocycle $\omega$ is a coboundary if there exists a linear map $\eta: \mathcal{L} \rightarrow \mathcal{L}$ such that

$$
\omega(x, y)=\left(d_{1} \eta\right)(x, y):=\eta([x, y])-[x, \eta(y)]-[\eta(x), y] .
$$

The vector space of coboundaries is denoted by $\mathrm{B}^{2}(\mathcal{L}, \mathcal{L})$ and is a subspace of $\mathrm{Z}^{2}(\mathcal{L}, \mathcal{L})$. The quotient space is the Lie algebra two-cohomology with values in the adjoint module, denoted by $\mathrm{H}^{2}(\mathcal{L}, \mathcal{L})$, see e.g. 11, 2] for further details.

Coming back to the deformation (2.1) we see that the Jacobi identity for the algebras $\mathcal{L}_{t}$ implies that the two-cochain $\phi_{1}$ is indeed a cocycle, i.e. it fulfills $d_{2} \phi_{1}=0$. If $\phi_{1}$ vanishes identically, the first nonvanishing $\phi_{i}$ will be a cocycle. If $\mu_{t}^{\prime}$ is an equivalent deformation (with cochains $\phi_{i}^{\prime}$ ) then

$$
\phi_{1}^{\prime}-\phi_{1}=d_{1} \psi_{1} .
$$

Hence, every equivalence class of deformations defines uniquely an element of $\mathrm{H}^{2}(\mathcal{L}, \mathcal{L})$. This class is called the differential of the deformation. The differential of a family which is equivalent to a trivial family will be the zero cohomology class.

\subsection{Global deformations.}

The deformation $\mathcal{L}_{t}$ might be considered not only as a family of Lie algebras, but also as a Lie algebra over the algebra $\mathbb{K}[[t]]$ of formal power series over $\mathbb{K}$. From this description a natural step is to allow $\mathbb{K}\left[\left[t_{1}, t_{2}, \ldots, t_{k}\right]\right]$, or an arbitrary commutative algebra $A$ over $\mathbb{K}$ with unit as the base of a deformation.

In the following we will assume that $A$ is a commutative algebra over $\mathbb{K}$ (where $\mathbb{K}$ is a field of characteristic zero) which admits an augmentation $\epsilon: A \rightarrow \mathbb{K}$. This says that $\epsilon$ is a $\mathbb{K}$-algebra homomorphism, e.g. $\epsilon\left(1_{A}\right)=1$. The ideal $m_{\epsilon}:=\operatorname{Ker} \epsilon$ is a maximal ideal of $A$. Vice versa, given a maximal ideal $m$ of $A$ with $A / m \cong \mathbb{K}$, the natural quotient map defines an augmentation.

If $A$ is a finitely generated $\mathbb{K}$-algebra over an algebraically closed field $\mathbb{K}$ then $A / m \cong \mathbb{K}$ is true for every maximal ideal $m$. Hence, in this case every such $A$ admits at least one augmentation and all maximal ideals are coming from augmentations.

Let us consider a Lie algebra $\mathcal{L}$ over the field $\mathbb{K}, \epsilon$ a fixed augmentation of $A$, and $m=\operatorname{Ker} \epsilon$ the associated maximal ideal.

Definition 2.1. (9]) A global deformation $\lambda$ of $\mathcal{L}$ with base $(A, m)$ or simply with base $A$, is a Lie $A$-algebra structure on the tensor product $A \otimes_{\mathbb{K}} \mathcal{L}$ with bracket $[.,]_{\lambda}$ such that

$$
\epsilon \otimes \mathrm{id}: A \otimes \mathcal{L} \rightarrow \mathbb{K} \otimes \mathcal{L}=\mathcal{L}
$$

is a Lie algebra homomorphism.

Specifically, it means that for all $a, b \in A$ and $x, y \in \mathcal{L}$,

(1) $[a \otimes x, b \otimes y]_{\lambda}=(a b \otimes \mathrm{id})[1 \otimes x, 1 \otimes y]_{\lambda}$,

(2) $[., .]_{\lambda}$ is skew-symmetric and satisfies the Jacobi identity,

(3) $\epsilon \otimes \mathrm{id}\left([1 \otimes x, 1 \otimes y]_{\lambda}\right)=1 \otimes[x, y]$. 
By Condition (1) to describe a deformation it is enough to give the elements $[1 \otimes x, 1 \otimes y]_{\lambda}$ for all $x, y \in \mathcal{L}$. From condition (3) it follows that for them the Lie product has the form

$$
[1 \otimes x, 1 \otimes y]_{\lambda}=1 \otimes[x, y]+\sum_{i} a_{i} \otimes z_{i},
$$

with $a_{i} \in m, z_{i} \in \mathcal{L}$.

A deformation is called trivial if $A \otimes_{\mathbb{K}} \mathcal{L}$ carries the trivially extended Lie structure, i.e. (2.7) reads as $[1 \otimes x, 1 \otimes y]_{\lambda}=1 \otimes[x, y]$. Two deformations of a Lie algebra $\mathcal{L}$ with the same base $A$ are called equivalent if there exists a Lie algebra isomorphism between the two copies of $A \otimes \mathcal{L}$ with the two Lie algebra structures, compatible with $\epsilon \otimes$ id.

We say that a deformation is local (in the algebraic sense) if $A$ is a local $\mathbb{K}$-algebra with unique maximal ideal $m_{A}$. By assumption $m_{A}=\operatorname{Ker} \epsilon$ and $A / m_{A} \cong \mathbb{K}$. In case that in addition $m_{A}^{2}=0$, the deformation is called infinitesimal.

An important class of examples is given if $A$ is the algebra of regular functions of an affine variety $V$, i.e. $A=\mathbb{K}[V]$. The algebra $A$ is also called the coordinate algebra of the affine variety $V$. Let us assume that $\mathbb{K}$ is algebraically closed. It is known that every finitely generated (as ring) $\mathbb{K}$-algebra which is reduced, i.e. which has no nilpotent elements, is the algebra of regular functions of a suitable affine variety. The variety might be reducible if $A$ has zero-divisors. The maximal ideals $m_{x}$ of $A$ correspond exactly to the points $x \in X$, and exactly to the set of augmentations $\epsilon_{x}$. Fixing an augmentation $\epsilon_{x_{0}}$ means fixing a point $x_{0} \in V$. If $A$ is a non-local ring, there will be different maximal ideals, hence also different augmentations. Let $\mathcal{L}$ be a $\mathbb{K}$-vector space and assume that there exists a Lie $A$-algebra structure $[., .]_{A}$ on $A \otimes_{\mathbb{K}} \mathcal{L}$. Given an augmentation $\epsilon: A \rightarrow \mathbb{K}$ with associated maximal ideal $m_{\epsilon}=\operatorname{Ker} \epsilon$, one obtains a Lie $\mathbb{K}$-algebra structure $\mathcal{L}^{\epsilon}=\left(\mathcal{L},[.,]_{\epsilon}\right)$ on the vector space $\mathcal{L}$ by passing to the quotient $A / m_{\epsilon}$. In this way the Lie algebra $A \otimes_{\mathbb{K}} \mathcal{L}$ gives a family of Lie algebra structures on the space $\mathcal{L}$ parameterized by the points of $V$. We obtain a map

$V \quad \rightarrow \quad\{$ set of Lie algebra structures on the vector space $\mathcal{L}\}$.

We will denote both $A=\mathbb{K}[V]$ and $V$ as the base of the deformation. It is quite convenient to consider both (the algebraic and the geometric) pictures. Clearly, the Lie $A$ algebra $A \otimes_{\mathbb{K}} \mathcal{L}$, i.e. the global family over $A$, is a deformation in the sense of Definition 2.1 with basis $\left(A, m_{x}\right)$ for all points $x \in V$.

If we choose a basis $\left\{T_{a}\right\}_{a \in J}$ of the Lie algebra $\mathcal{L}$, the Lie structure in $\mathcal{L}$ is given by the structure constants $\left\{C_{a, b}^{c}\right\}$ defined via

$$
\left[T_{a}, T_{b}\right]=\sum_{c \in J}{ }^{\prime} C_{a, b}^{c} T_{c}, \quad a, b \in J
$$

Here $\sum^{\prime}$ denotes, that only a finite number of the summands will be different from 0 . Assume that we have a deformation over $A=\mathbb{K}[V]$ in the sense of Definition 2.1] where $\mathcal{L}$ lies above $x_{0}$. The elements $1 \otimes T_{a}$ are now an $A$-basis of $A \otimes \mathcal{L}$ and Equation (2.7) can be written as

$$
\left[1 \otimes T_{a}, 1 \otimes T_{b}\right]_{\epsilon_{x}}=\sum_{c \in J}{ }^{\prime} C_{a, b}^{c}(x) 1 \otimes T_{c} \quad a, b \in J
$$

with algebraic functions

$$
C_{a, b}^{c}(x) \in \mathbb{K}[V], \quad \text { with } \quad\left(C_{a, b}^{c}(x)-C_{a, b}^{c}\right) \in m_{x_{0}} \text {, or equivalently } C_{a, b}^{c}\left(x_{0}\right)=C_{a, b}^{c} .
$$


Note that the range of the summation might be bigger than the original one. In this way we get back the intuitive picture of algebraically varying structure constants. The structure constants of the Lie algebra $\mathcal{L}_{x^{\prime}}$ lying above the point $x^{\prime}$ are given by $C_{a, b}^{c}\left(x^{\prime}\right)$.

A very import special case is a deformation over the affine line $\mathbb{A}^{1}$. Here the corresponding algebra is $A=\mathbb{K}\left[\mathbb{A}^{1}\right]=\mathbb{K}[t]$, the algebra of polynomials in one variable. For a deformation of the Lie algebra $\mathcal{L}=\mathcal{L}_{0}$ over the affine line, the Lie structure $\mathcal{L}_{\alpha}$ in the fiber over the point $\alpha \in \mathbb{K}$ is given by considering the augmentation corresponding to the maximal ideal $m_{\alpha}=(t-\alpha)$.

Remark. Despite the fact that we consider only deformations over affine varieties, we call this type of deformations global, as we allow also non-local algebras. Nevertheless, it might be useful to study deformations over more general global basis, like projective varieties, schemes, complex manifolds, analytic spaces, etc. As in this article we are only interested in rigidity questions, and they are geometrically local, i.e. only the case of open neighbourhoods (e.g. local affine neighbourhoods) is of importance, see Definition 2.5, the given definition will be sufficient for us.

For further reference we note

Definition 2.2. Let $A^{\prime}$ be another commutative algebra over $\mathbb{K}$ with a fixed augmentation $\epsilon^{\prime}: A^{\prime} \rightarrow \mathbb{K}$, and let $\phi: A \rightarrow A^{\prime}$ be an algebra homomorphism with $\phi(1)=1$ and $\epsilon^{\prime} \circ \phi=\epsilon$. If a deformation $\lambda$ of $\mathcal{L}$ with base $(A, \operatorname{Ker} \epsilon=m)$ is given, then the push-out $\lambda^{\prime}=\phi_{*} \lambda$ is the deformation of $\mathcal{L}$ with base $\left(A^{\prime}, \operatorname{Ker} \epsilon^{\prime}=m^{\prime}\right)$, and Lie algebra structure

$$
\left[a_{1}^{\prime} \otimes_{A}\left(a_{1} \otimes l_{1}\right), a_{2}^{\prime} \otimes_{A}\left(a_{2} \otimes l_{2}\right)\right]_{\lambda^{\prime}}:=a_{1}^{\prime} a_{2}^{\prime} \otimes_{A}\left[a_{1} \otimes l_{1}, a_{2} \otimes l_{2}\right]_{\lambda},
$$

$\left(a_{1}^{\prime}, a_{2}^{\prime} \in A^{\prime}, a_{1}, a_{2} \in A, l_{1}, l_{2} \in \mathcal{L}\right)$ on $A^{\prime} \otimes \mathcal{L}=\left(A^{\prime} \otimes_{A} A\right) \otimes \mathcal{L}=A^{\prime} \otimes_{A}(A \otimes \mathcal{L})$. Here $A^{\prime}$ is regarded as an $A$-module with the structure $a a^{\prime}=\phi(a) a^{\prime}$.

\subsection{Formal deformations.}

Let $A$ be a complete local algebra over $\mathbb{K}$, so $A=\overleftarrow{\lim }_{n \rightarrow \infty}\left(A / m^{n}\right)$, where $m$ is the maximal ideal of $A$. Furthermore, we will assume that $A / m \cong \mathbb{K}$, and $\operatorname{dim}\left(m^{k} / m^{k+1}\right)<\infty$ for all $k$.

Definition 2.3. A formal deformation of $\mathcal{L}$ with base $A$ is a Lie algebra structure on the completed tensor product $A \widehat{\otimes} \mathcal{L}=\overleftarrow{\lim }_{n \rightarrow \infty}\left(\left(A / m^{n}\right) \otimes \mathcal{L}\right)$ such that

$$
\epsilon \widehat{\otimes} \mathrm{id}: A \widehat{\otimes} \mathcal{L} \rightarrow \mathbb{K} \otimes \mathcal{L}=\mathcal{L}
$$

is a Lie algebra homomorphism.

If $A=\mathbb{K}[[t]]$, then a formal deformation of $\mathcal{L}$ with base $A$ is the same as a formal 1-parameter deformation of $\mathcal{L}$ (see [12]).

There is an analogous definition for equivalence of deformations parameterized by a complete local algebra. 


\subsection{The case of finite-dimensional Lie algebras.}

In this subsection let $\mathcal{L}$ be a finite-dimensional Lie algebra of dimension $n$ over $\mathbb{K}$. Let $\left\{T_{a}\right\}_{a=1, \ldots, n}$ be a set of basis elements for the vector space $V$ the Lie algebra $\mathcal{L}$ is modeled on. The Lie structure $\mu$ is fixed by the structure constants $\left\{C_{a, b}^{c}\right\}_{a, b, c=1, \ldots, n}$, defined by

$$
\left[T_{a}, T_{b}\right]=\sum_{c=1}^{n} C_{a, b}^{c} T_{c}, \quad a, b=1, \ldots, n .
$$

From the skew-symmetry and the Jacobi identity for the Lie algebra $\mathcal{L}$ it follows that the structure constants obey the following relations

$$
\begin{gathered}
C_{a, b}^{c}+C_{b, a}^{c}=0, \quad a, b, c=1, \ldots, n, \\
\sum_{l=1}^{n}\left(C_{a, b}^{l} C_{l, c}^{d}+C_{b, c}^{l} C_{l, a}^{d}+C_{c, a}^{l} C_{l, b}^{d}\right)=0, \quad a, b, c, d=1, \ldots, n .
\end{gathered}
$$

Conversely, given such a set of $\left\{C_{a, b}^{c}\right\}_{a, b, c=1, \ldots, n}$, satisfying (2.14) the vector space generated by $n$ basis elements $T_{a}$ carries the structure of a Lie algebra defined via (2.13).

Obviously, (2.14) are algebraic equations and the vanishing set $\operatorname{Lalg}_{n}$ in $\mathbb{K}^{N}, N=n^{3}$, of the ideal generated by them "parameterizes" the possible Lie algebra structures on the $n$-dimensional vector space $V$. Strictly speaking, it is more appropriate to talk about the scheme, as one should better consider the not necessarily reduced structure on $\operatorname{Lalg}_{n}$, see Rauch 24]. Furthermore, as $\mu$ is a bilinear map $V \times V \rightarrow V$, the structure constants might be considered more canonically as elements of $V^{*} \otimes V^{*} \otimes V$ with $V^{*}$ denoting the dual space of $V$.

Given a linear automorphism $\Phi \in \mathrm{GL}(V)$, it will define an action on $V^{*} \otimes V^{*} \otimes V$ by

$$
(\Phi \star \mu)(x, y)=\Phi\left(\mu\left(\Phi^{-1}(x), \Phi^{-1}(y)\right)\right),
$$

which respects the conditions defining a Lie algebra structure. The Lie algebras $(V, \mu)$ and $\left(V, \mu^{\prime}\right)$ will be isomorphic iff $\mu$ and $\mu^{\prime}$ are in the same orbit under the GL $(V)$ action. On the level of structure constants, i.e. after choosing a basis in $V$, we obtain a GL( $n)$ action on $\operatorname{Lalg}_{n}$. In this way the isomorphy classes of Lie algebras of dimension $n$ correspond exactly to the GL $(n)$ orbits of $\operatorname{Lalg}_{n}$.

For further reference we note, that a finite-dimensional Lie algebra $\mathcal{L}$ is called nonsingular if the corresponding point $\mu$ in the scheme $\operatorname{Lalg}_{n}$ is a non-singular point in the sense of algebraic geometry.

\subsection{Rigidity.}

Intuitively, rigidity of a Lie algebra $\mathcal{L}$ means that we cannot deform the Lie algebra. Or, formulated differently, given a family of Lie algebras containing $\mathcal{L}$ as the special element $\mathcal{L}_{0}$, any element $\mathcal{L}_{t}$ in the family "nearby" will be isomorphic to $\mathcal{L}_{0}$. Of course, the definition depends on the category in which the deformations are considered.

As "nearby" is already encoded in the infinitesimal and formal setting we can define:

Definition 2.4. (a) A Lie algebra $\mathcal{L}$ is infinitesimally rigid iff every infinitesimal deformation of it is equivalent to the trivial deformation.

(b) A Lie algebra $L$ is formally rigid iff every formal deformation of it is equivalent to the trivial deformation. 
For the global deformation such a condition would be too much to require as the following example shows.

Example. Let $\mathcal{L}$ be any non-abelian Lie algebra with Lie bracket [.,.]. We define a family of Lie algebras $\mathcal{L}_{t}$ over the algebra $\mathbb{K}[t]$ (i.e. geometrically over the affine line $\mathbb{K}$ ) by taking as Lie bracket the bracket $[x, y]_{t}:=(1-t)[x, y]$. If we set $t=0$ we obtain back our Lie algebra $\mathcal{L}$. Moreover, as long as $t \neq 1$ the algebras $\mathcal{L}_{t}$ are isomorphic to $\mathcal{L}$, but $\mathcal{L}_{1}$ as abelian Lie algebra will be non-isomorphic. Hence such a family will never be the trivial family. But if we restrict the family to the (Zariski) open subset $\mathbb{K} \backslash\{1\}$, of the base $\mathbb{K}$, we obtain a trivial family.

In the purely algebraic setting we can apply Zariski topology. The Zariski topology (Ztopology) of a ring $R$ is a topology on the set of prime ideals, yielding a topological space $\operatorname{Spec}(R)$. Instead of considering the general case, we will restrict ourselves to the situation which is of interest here. Let $\mathbb{K}$ be an algebraically closed field of characteristic zero and $A$ a finitely generated reduced $\mathbb{K}$-algebra. Recall that this corresponds geometrically to the case of affine varieties $V$ as base. A subset $W$ of $V$ is called Z-closed if it is the vanishing set of an ideal in $A=\mathbb{K}[V]$. The set $W$ itself will be an affine variety. A subset $U$ of $V$ is called Z-open if it is the complement of a closed set. A basis of the open set of the topology is given by Z-open affine varieties. These basis sets are open sets in $V$ and they themselves are affine (this says they are the vanishing sets of an ideal in a suitable affine space), see [14] for this and further results.

In particular, given an arbitrary Z-open subset $U$ containing the point $x \in V$ we can always find an open subset $W$ of $U$ which itself is affine. For simplicity (and in accordance with the general picture) let us use $\operatorname{Spec}(A)$ to denote the variety $V$ and let us identify the (closed) points in $V$ with the maximal ideals.

Definition 2.5. A Lie algebra $\mathcal{L}$ is algebraic-geometrically rigid of just geometrically rigid if for every deformation of $\mathcal{L}$ in the sense of Definition 2.1] with base $(A, m)$, where $A$ is a finitely generated reduced algebra over the algebraically closed field $\mathbb{K}$ and $m$ a maximal ideal, there exists a Zariski open affine neighbourhood $\operatorname{Spec}(B)$ of the point $m$ in $\operatorname{Spec}(A)$ such that the restricted family over $(B, m)$ is equivalent to the trivial family.

Note that the restriction given by the geometric picture $\operatorname{Spec}(B) \hookrightarrow \operatorname{Spec}(A)$ corresponds exactly to the push-out of the deformation given by the induced $\mathbb{K}$-algebra homomorphism $A \rightarrow B$. Note also, that with a slight abuse of notation we understand in $(B, m)$ by $m$ the ideal generated by the image in $B$ of the ideal $m$ of $A$.

In the case if the base field is $\mathbb{C}$ (or $\mathbb{R}$ ) and the base of the deformation is a finitedimensional analytic manifold $M$ with a chosen point $x_{0} \in M$ we consider also the usual topology on $M$. In particular, we can talk about open subsets of $M$ containing $x_{0}$.

Definition 2.6. A Lie algebra $\mathcal{L}$ over $\mathbb{C}($ or $\mathbb{R})$ is analytically rigid if for every family over a finite-dimensional analytic manifold $\left(M, x_{0}\right)$ with base point $x_{0}$, with special fiber $\mathcal{L} \cong \mathcal{L}_{x_{0}}$ over the point $x_{0} \in M$, there is an open neighbourhood $U$ of $x_{0}$, such that the restriction of this family is equivalent to the trivial family.

Obviously, as the analytic rigidity is a (geometrically) local condition, it is enough to establish rigidity by considering families over $\mathbb{C}^{k}$ (resp. $\left.\mathbb{R}^{k}\right)$. 
There are other definitions of rigidity by considering differentiable manifolds or analytic spaces as base. Moreover, we could incorporate infinite dimensional manifolds or nonNoetherian algebras as base of the deformations. But for the families discussed in this article we do not need them. Hence, we will not discuss them here. Already in the case that the Lie algebra is infinite dimensional and the base is finite-dimensional, interesting examples appear. It can be expected that the situation will become even more intricate by allowing infinite dimensional bases. To our knowledge not much is known in this direction, whereas the topic is surely interesting. We intend to come back to it in the future.

In case that the Lie algebra is finite-dimensional there is another important definition of rigidity.

Definition 2.7. (12, 22]) Let $\mathcal{L}$ be a Lie algebra of dimension $n$ corresponding to the point $\mu=\left\{C_{a, b}^{c}\right\}$ in the space of structure constants. The algebra $\mathcal{L}$ is called rigid (in the orbit sense) or just rigid if the orbit of $\left\{C_{a, b}^{c}\right\}$ under the $\mathrm{GL}(n)$ action is Zariski open in $\operatorname{Lalg}_{n}$.

Given a finite-dimensional Lie algebra $\mathcal{L}$ which is rigid (in the orbit sense) and a deformation over the base $\left(V, x_{0}\right)$, then the algebras $\mathcal{L}_{x}$ will be isomorphic to $\mathcal{L}_{x_{0}}=\mathcal{L}$ for any $\mathrm{x}$ in a Zariski open neighbouhood $U$ of $x_{0}$. Hence, (orbitally) rigidity implies geometric (and if $\mathbb{K}$ is equal $\mathbb{R}$ or $\mathbb{C}$ also analytic) rigidity. See Theorem 2.9 and Theorem 2.12 for partial inversions of this statement.

\subsection{Rigidity and cohomology.}

As explained in Section 2.1 there is a close connection between deformations of Lie algebras and the Lie algebra two-cohomology $\mathrm{H}^{2}(\mathcal{L}, \mathcal{L})$ with values in $\mathcal{L}$. The following is obvious.

Proposition 2.8. Let $\mathcal{L}$ be an arbitrary Lie algebra over $\mathbb{K}$, then $\mathcal{L}$ is infinitesimally rigid iff $\mathrm{H}^{2}(\mathcal{L}, \mathcal{L})=0$.

Theorem 2.9. (12], 21], 22]) Let $\mathcal{L}$ be a finite-dimensional Lie algebra. If $\mathrm{H}^{2}(\mathcal{L}, \mathcal{L})=0$ then the Lie algebra $\mathcal{L}$ is rigid in all the senses introduced above.

In particular, as infinitesimal rigidity is equivalent to $\mathrm{H}^{2}(\mathcal{L}, \mathcal{L})=0$, it already implies in finite dimension already rigidity in the formal, geometric, (and analytic) sense. This is definitely not true in infinite dimension, as we showed in [10] by exhibiting a deformation over the affine line $\mathbb{C}[t]$ of the Witt algebra which is non-trivial in every neighbourhood of $t=0$, despite the fact that the Witt algebra is infinitesimally and formally rigid. In Section 4 we will construct deformations of the current algebras $\overline{\mathfrak{g}}$ associated to finite dimensional complex Lie algebras $\mathfrak{g}$ which are neither geometrically nor analytically rigid despite that for $\mathfrak{g}$ simple the Lie algebra $\overline{\mathfrak{g}}$ is formally rigid [20].

Nevertheless, also in infinite dimensions we have the following theorem.

Theorem 2.10. (12], 21], 22]) Let $\mathcal{L}$ be an arbitrary Lie algebra. If $\mathrm{H}^{2}(\mathcal{L}, \mathcal{L})=0$ then $\mathcal{L}$ is formally rigid.

The theorem is also a corollary of the result of Fialowski [6] on the existence of versal formal families which will be recalled in the next subsection. 
For completeness and to contrast this with the infinite dimensional case let us quote the following results for the finite-dimensional case.

Theorem 2.11. (24]) Let $\mathcal{L}$ be a finite-dimensional Lie algebra corresponding to the point $\mu \in$ Lalg $_{n}$ then:

(a) The Zariski tangent space of the scheme Lalg $_{n}$ at the point $\mu$ can be naturally identified with $\mathrm{Z}^{2}(L, L)$.

(b) The Zariski tangent space of the GL( $n)$ orbit of $\mu$ (considered as reduced scheme) can be naturally identified with $\mathrm{B}^{2}(L, L)$.

From Theorem 2.11] and Theorem 2.9] and the fact that $\mathrm{H}^{2}(\mathcal{L}, \mathcal{L})=0$ implies that $\mu$ is a non-singular point [12, 22], the following is a consequence.

Theorem 2.12. Let $\mathcal{L}$ be a finite-dimensional Lie algebra. Then $\mathrm{H}^{2}(\mathcal{L}, \mathcal{L})=0$ iff $\mathcal{L}$ is rigid and is a nonsingular Lie algebra. Moreover, in this case all the above definitions of rigidity coincide.

Richardson [25] has given an example of a finite-dimensional Lie algebra with $\mathrm{H}^{2}(\mathcal{L}, \mathcal{L}) \neq$ 0 , which is nevertheless rigid in the orbit sense. Clearly, the $\mu$ corresponding to the Lie structure of this $\mathcal{L}$ is a singular point in $\operatorname{Lalg}_{n}$.

\subsection{Universal and versal deformations.}

As explained above and will be shown further down in this article, the strong relation between cohomology spaces and deformations over non-local rings breaks down in infinite dimension. It might be considered as a astonishing result that a tight connection still exists on the formal level.

Proposition 2.13. (9]) Assume that $\operatorname{dim} \mathrm{H}^{2}(\mathcal{L}, \mathcal{L})<\infty$, then there exists a universal infinitesimal deformation $\eta_{\mathcal{L}}$ of the Lie algebra $\mathcal{L}$ with base $B=\mathbb{K} \oplus \mathrm{H}^{2}(\mathcal{L}, \mathcal{L})^{*}$, where the second summand is the dual of $\mathrm{H}^{2}(\mathcal{L}, \mathcal{L})$ equipped with the zero multiplication, i.e.

$$
\left(\alpha_{1}, h_{1}\right) \cdot\left(\alpha_{2}, h_{2}\right)=\left(\alpha_{1} \alpha_{2}, \alpha_{1} h_{2}+\alpha_{2} h_{1}\right) .
$$

This means that for every infinitesimal deformation $\lambda$ of the Lie algebra $\mathcal{L}$ with finite dimensional base $A$, there exists a unique homomorphism $\phi: \mathbb{K} \oplus \mathrm{H}^{2}(\mathcal{L}, \mathcal{L})^{*} \rightarrow A$ such that $\lambda$ is equivalent to the push-out $\phi_{*} \eta_{\mathcal{L}}$.

Although in general it is impossible to construct a universal formal deformation, there is a so-called versal element.

Definition 2.14. ([5, 6] ) A formal deformation $\eta$ of $\mathcal{L}$ parameterized by a complete local algebra $\left(B, m_{B}\right)$ is called versal if for every deformation $\lambda$, parameterized by a complete local algebra $\left(A, m_{A}\right)$, there is a morphism $f: B \rightarrow A$ such that

1) The push-out $f_{*} \eta$ is equivalent to $\lambda$.

2) If $A$ satisfies $m_{A}^{2}=0$, then $f$ is unique.

Theorem 2.15. ([6], [9, Thm. 4.6]) Assume that $\operatorname{dim} \mathrm{H}^{2}(\mathcal{L}, \mathcal{L})<\infty$.

(a) There exists a versal formal deformation of $\mathcal{L}$.

(b) The base of the versal formal deformation is formally embedded into $\mathrm{H}^{2}(\mathcal{L}, \mathcal{L})$, i.e. it can be described in $\mathrm{H}^{2}(\mathcal{L}, \mathcal{L})$ by a finite system of formal equations. 
Hence if $\mathrm{H}^{2}(\mathcal{L}, \mathcal{L})=0$, every formal deformation will be equivalent to the trivial one, see Theorem 2.10 .

\subsection{Deformations of commutative algebras and Harrison cohomology.}

In this article we will deform current algebras by deforming associative and commutative algebras in a geometric way. The corresponding cohomology theory of such deformations is the Harrison cohomology 15 .

Let $\mathcal{A}$ be an associative and commutative algebra over $\mathbb{K}$. We only need here the space $\mathrm{H}_{\text {Harr }}^{2}(\mathcal{A}, \mathcal{A})$. Recall its definition. The two-cocycles are bilinear maps $F: \mathcal{A} \times \mathcal{A} \rightarrow \mathcal{A}$ such that

$$
\begin{gathered}
F(a, b)=F(b, a), \quad a, b \in \mathcal{A}, \\
\delta_{2} F(a, b, c):=a F(b, c)-F(a b, c)+F(a, b c)-F(a, b) c=0, \quad a, b, c \in \mathcal{A} .
\end{gathered}
$$

A two-cycle is a coboundary if there exists a linear map $\phi: \mathcal{A} \rightarrow \mathcal{A}$ such that

$$
F=\delta_{1} \phi(a, b)=a \phi(b)-\phi(a b)+\phi(a) b, \quad a, b \in \mathcal{A} .
$$

Note that $\mathrm{H}_{\text {Harr }}^{2}(\mathcal{A}, \mathcal{A})$ will be a subspace of the the Hochschild cohomology space $\mathrm{H}_{\text {Hoch }}^{2}(\mathcal{A}, \mathcal{A})$.

\section{KRICHEVER-NOVIKOV ALGEBRAS}

\subsection{The algebras with their almost-grading.}

Algebras of Krichever-Novikov type are generalizations of the Virasoro algebra, current algebras and all their related algebras. Let $M$ be a compact Riemann surface of genus $g$, or in terms of algebraic geometry, a smooth projective curve over $\mathbb{C}$. Let $N, K \in \mathbb{N}$ with $N \geq 2$ and $1 \leq K<N$. Fix

$$
I=\left(P_{1}, \ldots, P_{K}\right), \quad \text { and } \quad O=\left(Q_{1}, \ldots, Q_{N-K}\right)
$$

disjoint ordered tuples of distinct points ("marked points", "punctures") on the curve. In particular, we assume $P_{i} \neq Q_{j}$ for every pair $(i, j)$. The points in $I$ are called the in-points, the points in $O$ the out-points. Sometimes we consider $I$ and $O$ simply as sets and denote $A=I \cup O$ as a set.

Here we will need the following algebras. Let $\mathcal{A}$ be the associative algebra of those meromorphic functions on $M$ which are holomorphic outside the set of points $A$ with point-wise multiplication. Let $\mathcal{L}$ be the Lie algebra of meromorphic vector fields which are holomorphic outside of $A$ with the usual Lie bracket of vector fields. The algebra $\mathcal{L}$ is called the vector field algebra of Krichever-Novikov type. They were introduced and their structure was studied by Krichever and Novikov [18. The corresponding generalization to the multi-point case was done in [27, 28, 29, 30. Obviously, both $\mathcal{A}$ and $\mathcal{L}$ are infinite dimensional algebras.

Furthermore we will need the higher-genus, multi-point current algebra of KricheverNovikov type. We start with $\mathfrak{g}$ a complex finite-dimensional Lie algebra and endow the tensor product $\overline{\mathcal{G}}=\mathfrak{g} \otimes_{\mathbb{C}} \mathcal{A}$ with the Lie bracket

$$
[x \otimes f, y \otimes g]=[x, y] \otimes f \cdot g, \quad x, y \in \mathfrak{g}, \quad f, g \in \mathcal{A} .
$$

The algebra $\overline{\mathcal{G}}$ is the higher genus current algebra. It is an infinite dimensional Lie algebra and might be considered as the Lie algebra of $\mathfrak{g}$-valued meromorphic functions on the Riemann surface with poles only outside of $A$. 
The classical genus zero and $N=2$ point case is give by the geometric data

$$
M=\mathbb{P}^{1}(\mathbb{C})=S^{2}, \quad I=\{z=0\}, \quad O=\{z=\infty\} .
$$

In this case the algebras are the well-known algebras of Conformal Field Theory (CFT). For the function algebra we obtain $\mathcal{A}=\mathbb{C}\left[z^{-1}, z\right]$, the algebra of Laurent polynomials. The vector field algebra $\mathcal{L}$ is the Witt algebra generated by $l_{n}=z^{n+1} \frac{d}{d z}, n \in \mathbb{Z}$ with Lie bracket $\left[l_{n}, l_{m}\right]=(m-n) l_{n+m}$, and the current algebra $\overline{\mathcal{G}}$ is the standard current algebra $\overline{\mathfrak{g}}=\mathfrak{g} \otimes \mathbb{C}\left[z^{-1}, z\right]$ with Lie bracket

$$
\left[x \otimes z^{n}, y \otimes z^{m}\right]=[x, y] \otimes z^{n+m} \quad x, y, \quad n, m \in \mathbb{Z} .
$$

For infinite dimensional algebras, modules and their representation theory a graded structure is usually important to obtain structure results. In fact, to define certain types of representations which are fundamental, e.g. in CFT like the highest weight representations, one uses grading.

In the classical situation the algebras are obviously graded by taking as degree $\operatorname{deg} l_{n}:=$ $n$ and $\operatorname{deg} x \otimes z^{n}:=n$. For higher genus there is usually no grading. But it was observed by Krichever and Novikov in the two-point case that a weaker concept, an almost-graded structure, will be enough to develop an interesting theory of representations (Verma modules, etc.).

Definition 3.1. Let $\mathcal{A}$ be an (associative or Lie) algebra admitting a direct decomposition as vector space $\mathcal{A}=\bigoplus_{n \in \mathbb{Z}} \mathcal{A}_{n}$. The algebra $\mathcal{A}$ is called an almost-graded algebra if (1) $\operatorname{dim} \mathcal{A}_{n}<\infty$ and (2) there are constants $R$ and $S$ such that

$$
\mathcal{A}_{n} \cdot \mathcal{A}_{m} \subseteq \bigoplus_{h=n+m+R}^{n+m+S} \mathcal{A}_{h}, \quad \forall n, m \in \mathbb{Z} .
$$

The elements of $\mathcal{A}_{n}$ are called homogeneous elements of degree $n$.

By exhibiting a special basis, for the multi-point situation such an almost grading was introduced in [27, 28, 29, 30. Essentially, this is done by fixing the order of the basis elements at the points in $I$ and $O$ in a complementary way to make them unique. In this way we obtain e.g. for the function algebra (resp. for the vector field algebra) basis elements $A_{n, p}$ (resp. $e_{n, p}$ ) with $n \in \mathbb{Z}$ and $p=1, \ldots, K$. As definition for the degree we take $(x \in \mathfrak{g})$

$$
\operatorname{deg}\left(e_{n, p}\right):=\operatorname{deg}\left(A_{n, p}\right):=\operatorname{deg}\left(x \otimes A_{n, p}\right):=n .
$$

In the following we will give an explicit description of the basis elements for those genus zero and one situation we need. Hence, we will not recall their general definition but only refer to the above quoted articles.

Proposition 3.2. [27, 30] With respect to the grading introduced by (3.5) the algebras $\mathcal{L}, \mathcal{A}$, and $\overline{\mathcal{G}}$ are almost-graded. The almost-grading depends on the splitting $A=I \cup O$. 


\subsection{Central extensions.}

In the construction of infinite dimensional representations of these algebras with certain desired properties (generated by a vacuum, irreducibility, unitarity, etc.) one is typically forced to "regularize" a "naive" action to make it well-defined. Important examples in CFT are the fermionic Fock space representations which are constructed by taking semiinfinite forms of a fixed weight.

From the mathematical point of view, with the help of a prescribed procedure one modifies the action to make it well-defined, but on the other hand, accepting that the modified action in compensation will be only a projective Lie action. Such projective actions are honest Lie actions for a suitable centrally extended algebra. In the classical case they are well-known. The unique non-trivial (up to equivalence and rescaling) central extension of the Witt algebra is the Virasoro algebra. For the current algebra $\mathfrak{g} \otimes \mathbb{C}\left[z^{-1}, z\right]$ if $\mathfrak{g}$ is a simple Lie algebra with Cartan-Killing form $\beta$, it is the corresponding affine Lie algebra $\widehat{\mathfrak{g}}$ (or, untwisted affine Kac-Moody algebra):

$$
\left[x \otimes z^{n}, y \otimes z^{m}\right]=[x, y] \otimes z^{n+m}-\beta(x, y) \cdot n \cdot \delta_{m}^{-n} \cdot t, \quad[t, \widehat{\mathfrak{g}}]=0, \quad x, y \in \mathfrak{g}, n, m \in \mathbb{Z} .
$$

For the extension to higher genus and many points the objects have to be "geometrized". First recall that for a Lie algebra $\mathcal{V}$ central extensions are classified (up to equivalence) by the second Lie algebra cohomology $\mathrm{H}^{2}(\mathcal{V}, \mathbb{C})$ of $\mathcal{V}$ with values in the trivial module $\mathbb{C}$. A bilinear form $\psi: \mathcal{V} \times \mathcal{V} \rightarrow \mathbb{C}$ is called a Lie algebra 2-cocycle iff $\psi$ is antisymmetric and fulfills the cocycle condition

$$
0=d_{2} \psi(x, y, z):=\psi([x, y], z)+\psi([y, z], x)+\psi([z, x], y) .
$$

To obtain central extensions of $\overline{\mathcal{G}}=\mathfrak{g} \otimes \mathcal{A}$ we start with $\mathfrak{g}$ being an arbitrary finitedimensional Lie algebra and $\beta$ a symmetric, invariant, bilinear form on it (not necessarily non-degenerate). Invariance means that we have $\beta([x, y], z)=\beta(x,[y, z])$ for all $x, y, z \in \mathfrak{g}$. We set $\widehat{\mathcal{G}}=\mathbb{C} \oplus \overline{\mathcal{G}}$ as vector space and introduce the bracket

$$
\widehat{[x \otimes f}, \widehat{y \otimes g}]=[x, \widehat{y] \otimes(} f g)+\beta(x, y) \frac{1}{2 \pi \mathrm{i}} \int_{C_{S}} f d g \cdot t, \quad[t, \widehat{\mathcal{G}}]=0 .
$$

Here we used for short $\widehat{x \otimes f}:=(0, x \otimes f), t:=(1,0)$ and $C_{S}$ denotes a cycle separating the points in $I$ from the points in $O$.

Proposition 3.3 (34]). The term $\beta(x, y) \frac{1}{2 \pi \mathrm{i}} \int_{C_{S}} f d g$ is a Lie algebra 2-cocycle of $\overline{\mathcal{G}}$ with values in the trivial module $\mathbb{C}$. Hence, the vector space $\widehat{\mathcal{G}}$ with structure (3.8) is a Lie algebra which defines a central extension of $\overline{\mathcal{G}}$.

These algebras are called higher genus (multi-point) affine algebras (of Krichever-Novikov type). In the classical situation (3.2) we obtain back (3.6). In the following we will use the term classical current algebra to denote the current algebra (3.6). In the new terminology it is a genus zero and two-point current algebra.

Note that the cocycle can be calculated as

$$
\beta(x, y) \cdot \frac{1}{2 \pi \mathrm{i}} \int_{C_{S}} f d g=\beta(x, y) \cdot \sum_{k=1}^{K} \operatorname{res}_{P_{k}}(f d g)=-\beta(x, y) \cdot \sum_{l=1}^{N-K} \operatorname{res}_{Q_{l}}(f d g) .
$$


As in general a non-vanishing $\beta$ is not uniquely given (up to rescaling), the central extension $\widehat{\mathcal{G}}$ will depend on it. Even in the case when $\mathfrak{g}$ is a simple Lie algebra, which implies that there is essentially only one non-vanishing form $\beta$, the Cartan-Killing form, for the higher genus or/and multi-point situation $\mathrm{H}^{2}(\overline{\mathcal{G}}, \mathbb{C})$ will be more than one-dimensional (e.g. take another path of integration which is not homologous to $C_{S}$ ). But the following is shown in 34 .

Theorem 3.4. Let $\mathfrak{g}$ be a simple finite-dimensional Lie algebra, then up to equivalence and multiplication with a scalar there is a unique non-trivial 2-cocycle class for the current algebra $\overline{\mathcal{G}}=\mathfrak{g} \otimes \mathcal{A}$ which has a "local" representative, i.e. which allows to extend the almost grading of $\overline{\mathcal{G}}$ to the central extension $\widehat{\mathcal{G}}$ defined by the local representative by assigning to the central element $t$ a degree. This class is given by (3.8) with $\beta$ being the Cartan-Killing form.

Also, in 34] a thorough treatment for the case when $\mathfrak{g}$ is semi-simple or even reductive can be found. Corresponding uniqueness results for almost-graded central extensions of $\mathcal{A}$ and $\mathcal{L}$ are shown in 33 .

\section{Current algebras for the elliptic curve Case}

In the following we will construct global deformations of the classical current algebra $\overline{\mathfrak{g}}=\mathfrak{g} \otimes \mathbb{C}\left[z^{-1}, z\right]$ and its central extension $\widehat{\mathfrak{g}}$. First we construct a family of associative algebras which contain the algebra of Laurent polynomials $\mathbb{C}\left[z^{-1}, z\right]$ as special element. The deformation family for the current algebra will be obtained by tensoring $\mathfrak{g}$ with this family. These families will be of geometric origin. More precisely, each non-special element in the family will be a current algebra of Krichever-Novikov type for the genus one (i.e. the elliptic) case. The construction of these families will not make any assumption about the finite-dimensional Lie algebra. Only later we will require $\mathfrak{g}$ to be a simple Lie algebra to contrast the existence of these global deformations which are (geometrically) locally not equivalent to trivial deformations, with the formal rigidity of $\overline{\mathfrak{g}}[20$.

\subsection{The family of elliptic curves.}

For the convenience of the reader we will recall the geometric picture from [10. As we have geometric degenerations in mind, it is more convenient to pass from the complex analytic picture (i.e. the language of Riemann surfaces) to the algebraic geometric picture (i.e. the language of curves). Every compact Riemann surface of genus one corresponds to an elliptic curve in the projective plane. Recall that the elliptic curves can be given as sets of solutions of the polynomial equation

$$
Y^{2} Z=4 X^{3}-g_{2} X Z^{2}-g_{3} Z^{3}, \quad g_{2}, g_{3} \in \mathbb{C}, \quad \text { with } \Delta:=g_{2}{ }^{3}-27 g_{3}{ }^{2} \neq 0 .
$$

Here $g_{2}$ and $g_{3}$ are parameterizing the individual curve and the condition $\Delta \neq 0$ assures that the curve will be nonsingular. Instead of (4.11) we can use the description

$$
Y^{2} Z=4\left(X-e_{1} Z\right)\left(X-e_{2} Z\right)\left(X-e_{3} Z\right)
$$

with

$$
e_{1}+e_{2}+e_{3}=0, \quad \text { and } \quad \Delta=16\left(e_{1}-e_{2}\right)^{2}\left(e_{1}-e_{3}\right)^{2}\left(e_{2}-e_{3}\right)^{2} \neq 0
$$


These presentations are related via

$$
g_{2}=-4\left(e_{1} e_{2}+e_{1} e_{3}+e_{2} e_{3}\right), \quad g_{3}=4\left(e_{1} e_{2} e_{3}\right) .
$$

The elliptic modular parameter classifying elliptic curves up to isomorphy is given as

$$
j=1728 \frac{g_{2}^{3}}{\Delta} .
$$

We set

$$
B:=\left\{\left(e_{1}, e_{2}, e_{3}\right) \in \mathbb{C}^{3} \mid e_{1}+e_{2}+e_{3}=0, \quad e_{i} \neq e_{j} \text { for } i \neq j\right\} .
$$

Inside the product $B \times \mathbb{P}^{2}$ we consider the family of elliptic curves $\mathcal{E}$ over $B$ defined via (4.2), with its obvious projection $\mathcal{E} \rightarrow B$. The family can be extended to

$$
\left.\widehat{B}:=\left\{e_{1}, e_{2}, e_{3}\right) \in \mathbb{C}^{3} \mid e_{1}+e_{2}+e_{3}=0\right\} .
$$

The fibers above $\widehat{B} \backslash B$ are singular cubic curves. Resolving the linear relation in $\widehat{B}$ via $e_{3}=-\left(e_{1}+e_{2}\right)$ we obtain a family over $\mathbb{C}^{2}$.

Consider the following complex lines in $\mathbb{C}^{2}$

$$
D_{s}:=\left\{\left(e_{1}, e_{2}\right) \in \mathbb{C}^{2} \mid e_{2}=s \cdot e_{1}\right\}, \quad s \in \mathbb{C}, \quad D_{\infty}:=\left\{\left(0, e_{2}\right) \in \mathbb{C}^{2}\right\} .
$$

Set also

for the punctured line. Now

$$
D_{s}^{*}=D_{s} \backslash\{(0,0)\}
$$

$$
B \cong \mathbb{C}^{2} \backslash\left(D_{1} \cup D_{-1 / 2} \cup D_{-2}\right) .
$$

Note that above $D_{1}^{*}$ we have $e_{1}=e_{2} \neq e_{3}$, above $D_{-1 / 2}^{*}$ we have $e_{2}=e_{3} \neq e_{1}$, and above $D_{-2}^{*}$ we have $e_{1}=e_{3} \neq e_{2}$. In all these cases we obtain a nodal cubic. Every nodal cubic $E_{N}$ can be given as

$$
Y^{2} Z=4(X-e Z)^{2}(X+2 e Z)
$$

where $e$ denotes the value of the coinciding $e_{i}=e_{j}(-2 e$ is then necessarily the remaining one). The singular point is the point $(e: 0: 1)$. It is a node.

Above the unique common intersection point $(0,0)$ of all $D_{s}$ there is the cuspidal cubic $E_{C}$ :

$$
Y^{2} Z=4 X^{3} \text {. }
$$

The singular point is $(0: 0: 1)$. In both cases the complex projective line is the desingularisation of the singular curve.

In all cases (non-singular or singular) the point $\infty=(0: 1: 0)$ lies on the curves. It is the only intersection with the line at infinity, and is a non-singular point. In passing to the affine plane in the following we will loose nothing. The affine curve will be given as the solution set of

$$
Y^{2}=4\left(X-e_{1}\right)\left(X-e_{2}\right)\left(X-e_{3}\right) .
$$

For the curves above the points in $D_{s}^{*}$ we calculate $e_{2}=s e_{1}$ and $e_{3}=-(1+s) e_{1}$ (resp. $e_{3}=-e_{2}$ if $\left.s=\infty\right)$. Due to the homogeneity, the modular parameter $j$ for the curves above $D_{s}^{*}$ will be constant along the line. In particular, the curves in the family lying above $D_{s}^{*}$ will be isomorphic. Their modular parameter calculates to

$$
j(s)=1728 \frac{4\left(1+s+s^{2}\right)^{3}}{(1-s)^{2}(2+s)^{2}(1+2 s)^{2}}, \quad j(\infty)=1728 .
$$




\subsection{The family of current algebras.}

First we define a family of function algebras $\mathcal{A}$ on these elliptic curves. We introduce the points where poles are allowed. For our purpose it is enough to consider two marked points. More marked points are considered in [31, [26]. We will always put one marking to $\infty=(0: 1: 0)$ and the other one to the point with the affine coordinate $\left(e_{1}, 0\right)$. These markings define two sections of the family $\mathcal{E}$ over $\widehat{B} \cong \mathbb{C}^{2}$. With respect to the group structure on the elliptic curve given by $\infty$ as the neutral element (the first marking), the second marking chooses a two-torsion point. All other choices of two-torsion points will yield isomorphic situations.

Proposition 4.1. For every elliptic curve $E_{\left(e_{1}, e_{2}\right)}$ over $\left(e_{1}, e_{2}\right) \in \mathbb{C}^{2} \backslash\left(D_{1}^{*} \cup D_{-1 / 2}^{*} \cup D_{-2}^{*}\right)$ the associative algebra $\mathcal{A}_{\left(e_{1}, e_{2}\right)}$ of functions on $E_{\left(e_{1}, e_{2}\right)}$ has a basis $\left\{A_{n}, n \in \mathbb{Z}\right\}$ such that the algebra structure is given as

$$
A_{n} \cdot A_{m}= \begin{cases}A_{n+m}, & \text { for } n \text { or } m \text { even }, \\ A_{n+m}+3 e_{1} A_{n+m-2} & \\ +\left(e_{1}-e_{2}\right)\left(2 e_{1}+e_{2}\right) A_{n+m-4}, & \text { for } n \text { and } m \text { both odd } .\end{cases}
$$

By setting $\operatorname{deg}\left(A_{n}\right):=n$, we obtain an almost-grading.

Proof. In 31] it was shown that a basis of the corresponding Krichever-Novikov function algebra $\mathcal{A}$ is given by

$$
A_{2 k}:=\left(X-e_{1}\right)^{k}, \quad A_{2 k+1}:=1 / 2 Y\left(X-e_{1}\right)^{k-1} \quad k \in \mathbb{Z} .
$$

The calculation of the structure constants is straightforward. In the case when both $n$ and $m$ are odd, one replaces $Y^{2}$ by $4\left(X-e_{1}\right)\left(X-e_{2}\right)\left(X-e_{3}\right)$ and uses $e_{1}+e_{2}+e_{3}=0$. The almost-grading is obvious.

The algebras in Proposition 4.1] defined with the structure (4.15) make sense also for the points $\left(e_{1}, e_{2}\right) \in D_{1} \cup D_{-1 / 2} \cup D_{-2}$. Altogether this defines a two-dimensional family of algebras parameterized over the affine plane $\mathbb{C}^{2}$, or described algebraically, over the polynomial algebra $\mathbb{C}\left[e_{1}, e_{2}\right]$. For $\left(e_{1}, e_{2}\right)=(0,0)$ we obtain the algebra of Laurent polynomials, if we make the identification $A_{n}=z^{n}$.

By tensoring this family with $\mathfrak{g}$ we get the following.

Theorem 4.2. Let $\mathfrak{g}$ be a finite-dimensional Lie algebra, and $V$ a vector space with basis $\left\{A_{n} \mid n \in \mathbb{Z}\right\}$ then for given complex values $e_{1}, e_{2}$ the vector space $\mathfrak{g} \otimes V$ carries the structure of a Lie algebra given by

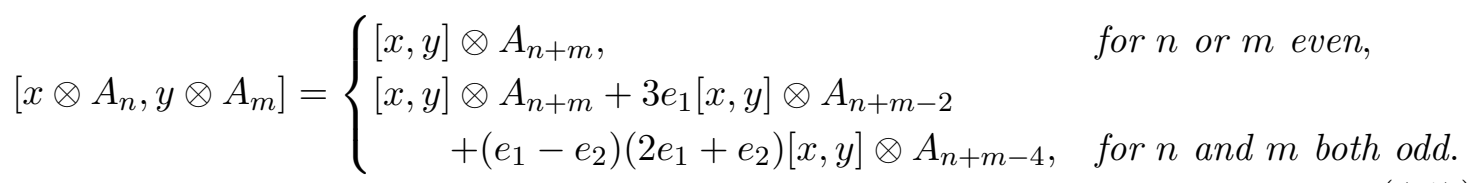

Here $x$ and $y$ are elements of $\mathfrak{g}$. In particular, these algebras define a two-parameter family of deformations of the current algebra $\overline{\mathfrak{g}}$, such that $\overline{\mathfrak{g}}$ corresponds to the point $(0,0)$ and the algebra over $\left(e_{1}, e_{2}\right) \in B$ corresponds to the elliptic affine algebra $\overline{\mathcal{G}}_{\left(e_{1}, e_{2}\right)}$ fixed by the geometric data. 
Further down we will identify the algebras over the other places. But first we study the family of algebras obtained by taking as base variety the (affine) line $D_{s}$ (for any $s$ ).

Corollary 4.3. For every $s \in \mathbb{C} \cup\{\infty\}$ the families of Theorem 4.2 define by restriction one-dimensional families of Lie algebras $\overline{\mathcal{G}}_{(e)}^{s}$ which are deformations of the classical current algebra $\overline{\mathfrak{g}}$ (corresponding to $e=0$ ) over the affine line $\mathbb{C}$, resp. the algebra $\mathbb{C}[e]$. For $s \neq \infty$ the family is given by

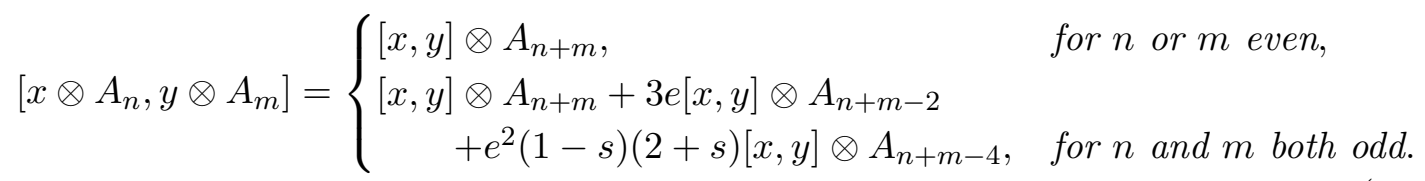

For $s=\infty$ the family is given by

$$
\left[x \otimes A_{n}, y \otimes A_{m}\right]= \begin{cases}{[x, y] \otimes A_{n+m},} & \text { for } n \text { or } m \text { even }, \\ {[x, y] \otimes A_{n+m}-e[x, y] \otimes A_{n+m-4},} & \text { for } n \text { and } m \text { both odd } .\end{cases}
$$

In the case when $s \neq 1,-1 / 2$ or 2 the algebras $\overline{\mathcal{G}}_{(e)}^{s}$ are elliptic current algebras. In any case for fixed $s$ we have $\overline{\mathcal{G}}_{(e)}^{s} \cong \overline{\mathcal{G}}_{\left(e^{\prime}\right)}^{s}$ as long as both $e, e^{\prime} \neq 0$.

Proof. If $s \neq \infty$ then $e_{2}=s e_{1}$ and (4.17) reduces to (4.18) if we set $e:=e_{1}$. For $s=\infty$ we have $e_{1}=0$ and obtain (4.19) if we set $e:=e_{2}^{2}$. If we rescale the basis elements $A_{n}^{*}=(\sqrt{e})^{-n} A_{n}$ (for $s \neq \infty$ ), we obtain for $e \neq 0$ always the algebra with $e=1$ in our structure equations. For $s=\infty$ a rescaling $(\sqrt[4]{e})^{-n} A_{n}$ will do the same (for $e \neq 0$ ). Hence we see that for fixed $s$ in all cases the algebras will be isomorphic above every point in $D_{s}$, as long as we are not above $(0,0)$.

The generic isomorphy class of the family $\overline{\mathcal{G}}_{(e)}^{s}$ can be given by taking $\overline{\mathcal{G}}_{(1)}^{s}$. To avoid cumbersome notation we often denote this isomorphy type just by $\overline{\mathcal{G}}^{s}$. But we have to keep in mind that by Proposition 4.7 the algebra $\overline{\mathcal{G}}^{s}$ will not be isomorphic to the special element $\overline{\mathcal{G}}_{(0)}^{s}=\overline{\mathfrak{g}}$.

Clearly, if we do the same kind of restrictions to $D_{s}$ for the families of associative algebras $\mathcal{A}_{\left(e_{1}, e_{2}\right)}$, we obtain similar one-dimensional algebraic families of commutative and associative algebras which deform the algebra of Laurent polynomials. We will denote these families by $\mathcal{A}_{(e)}^{s}$. Again for fixed $s$ all algebras over $e \neq 0$ will be isomorphic and we will denote this isomorphy type simply by $\mathcal{A}^{s}$. Proposition 4.6 will show that they are not isomorphic to the special element, the algebra of Laurent polynomial.

\subsection{The three point case for $\mathbb{P}^{1}$.}

There is another geometric family of algebras around. Its geometric picture is the three point situation for the Riemann sphere (the projective line). Because we will need these algebras later on anyhow, we will also give this family. The geometric situation is $M=\mathbb{P}^{1}(\mathbb{C}), I=\{\alpha,-\alpha\}$ and $O=\{\infty\}, \alpha \neq 0$. Let us denote the corresponding Krichever-Novikov function algebra by $\mathcal{V}_{(\alpha)}$. 
Proposition 4.4. For every $\alpha \in \mathbb{C}^{*}$ the algebra $\mathcal{V}_{(\alpha)}$ has a basis $\left\{A_{n} \mid n \in \mathbb{Z}\right\}$ such that its structure is given by

$$
A_{n} \cdot A_{m}= \begin{cases}A_{n+m}, & \text { for } n \text { or } m \text { even }, \\ A_{n+m}+\alpha^{2} A_{n+m-2}, & \text { for } n \text { and } m \text { both odd. }\end{cases}
$$

By setting $\operatorname{deg}\left(A_{n}\right):=n$ the algebra becomes an almost-graded algebra.

Proof. In 31] it was shown that a basis of (the vector space) $\mathcal{V}_{(\alpha)}$ is given by

$$
A_{2 k}:=(z-\alpha)^{k}(z+\alpha)^{k}, \quad A_{2 k+1}:=z(z-\alpha)^{k}(z+\alpha)^{k}, \quad k \in \mathbb{Z} .
$$

Here $z$ is the quasi-global coordinate on $\mathbb{P}^{1}(\mathbb{C})$. The structure follows by direct calculations.

Again, the algebra structure makes also sense for $\alpha=0$. In this case one obtains the algebra of Laurent polynomials.

For the corresponding current algebra $\mathcal{Y}_{(\alpha)}:=\mathfrak{g} \otimes \mathcal{V}_{(\alpha)}$ we obtain the following.

Theorem 4.5. There exists a one-dimensional family of current algebras $\mathcal{Y}_{(\alpha)}$ with Lie structure

$$
\left[x \otimes A_{n}, y \otimes A_{m}\right]= \begin{cases}{[x, y] \otimes A_{n+m},} & \text { for } n \text { or } m \text { even }, \\ {[x, y] \otimes A_{n+m}+\alpha^{2}[x, y] \otimes A_{n+m-2},} & \text { for } n \text { and } m \text { both odd },\end{cases}
$$

which is a deformation of the classical current algebra $\overline{\mathfrak{g}}$. Moreover, $\mathcal{Y}_{(\alpha)}$ is equivalent to the families $\overline{\mathcal{G}}_{(e)}^{1}$ and $\overline{\mathcal{G}}_{(e)}^{-2}$.

In Section 5 we will give a geometric explanation for the latter identification.

\subsection{Isomorphy question.}

In this subsection we will show that the current algebras $\overline{\mathcal{G}}^{(s)}$ are not isomorphic to the classical current algebra $\overline{\mathfrak{g}}$ if $\mathfrak{g}$ is semi-simple. Hence, the families introduced are nontrivial algebraic-geometric deformations for the (formally) rigid classical current algebra $\overline{\mathfrak{g}}$ (in case when $\mathfrak{g}$ is simple).

Proposition 4.6. The algebras $\mathcal{A}^{s}$ are not isomorphic to the algebra of Laurent polynomials.

Proof. The involved algebras are the algebras of meromorphic functions on projective curves with at least one point removed. Such curves are affine curves and these algebras are the affine coordinate algebras (i.e the algebras of regular functions) of these curves. The isomorphy class of the coordinate algebra is uniquely given by the isomorphy class of the affine curve, e.g. see [14. Any algebra $\mathcal{A}^{s}$ for $s \neq 1,-2,-1 / 2$ corresponds to an elliptic curve with two points removed, both algebras $\mathcal{A}^{1}$ and $\mathcal{A}^{-2}$ correspond to the projective line with three points removed, and $\mathcal{A}^{-1 / 2}$ corresponds to the nodal cubic with two (nonsingular) points removed. But the algebra of Laurent polynomials corresponds to the projective line with two points removed. The affine curves are not isomorphic as can easily be seen from the fact that their fundamental groups are different. Hence, also the algebras are not isomorphic. 
Proposition 4.7. Let $\mathfrak{g}$ be a semi-simple finite-dimensional Lie algebra, and $\mathcal{A}$ and $\mathcal{B}$ two associative, commutative algebras (with units). If the current algebras $\mathfrak{g} \otimes \mathcal{A}$ and $\mathfrak{g} \otimes \mathcal{B}$ are isomorphic as Lie algebras then $\mathcal{A}$ and $\mathcal{B}$ are isomorphic as associative algebras.

Proof. Let $\mathfrak{g}$ be a semi-simple finite-dimensional Lie algebra, and $P: \mathfrak{g} \otimes \mathcal{A} \rightarrow: \mathfrak{g} \otimes \mathcal{B}$ a Lie isomorphism of the current algebras. The Lie algebra $\mathfrak{g}$ admits a $s l(2)$ subalgebra and hence also $\mathfrak{g} \otimes \mathcal{A}$ admits a $\operatorname{sl}(2) \otimes \mathcal{A}$ subalgebra. By restriction we obtain a Lie isomorphism $P: \operatorname{sl}(2) \otimes \mathcal{A} \rightarrow P(s l(2) \otimes \mathcal{A})$. Via $\operatorname{sl}(2) \cong \operatorname{sl}(2) \otimes 1_{A}\left(1_{A}\right.$ the unit in $\left.\mathcal{A}\right)$, $s l(2)$ is a subalgebra of $s l(2) \otimes \mathcal{A}$. Denote by $h, e, f$ the standard generators of $s l(2)$, i.e.

$$
h=\left(\begin{array}{cc}
1 & 0 \\
0 & -1
\end{array}\right), e=\left(\begin{array}{ll}
0 & 1 \\
0 & 0
\end{array}\right), f=\left(\begin{array}{ll}
0 & 0 \\
1 & 0
\end{array}\right), \quad[h, e]=2 e,[h, f]=-2 f,[e, f]=h .
$$

The image $P\left(s l(2) \otimes 1_{A}\right)$ is isomorphic to $s l(2)$. In particular, $P\left(h \otimes 1_{A}\right)$ will be mapped to a basis of its Cartan subalgebra. After applying an inner automorphism, we can assume that $P\left(h \otimes 1_{A}\right)=h \otimes a_{1}$ with $a_{1} \in B, a_{1} \neq 0$. Let

$$
P\left(e \otimes 1_{A}\right)=h \otimes b_{1}+e \otimes b_{2}+f \otimes b_{3}, \quad P\left(f \otimes 1_{A}\right)=h \otimes c_{1}+e \otimes c_{2}+f \otimes c_{3},
$$

with $b_{i}, c_{i} \in \mathcal{B}, i=1,2,3$. Using the structure equations above we see that there exist only two solutions: (A) $a_{1}=1, b_{2}=\alpha$ with an invertible element $\alpha \in \mathcal{B}, c_{3}=\alpha^{-1}$, and all other elements equal zero; and (B) $a_{1}=-1, b_{3}=\alpha$ with an invertible element $\alpha \in \mathcal{B}, c_{2}=\alpha^{-1}$, and all other elements equal zero. After an inner automorphism (given by $\left.\left(\begin{array}{cc}0 & 1 \\ -1 & 0\end{array}\right)\right)$ the solution (B) is transferred to solution (A). Hence, we can assume that $P$ has the property

$$
P\left(h \otimes 1_{A}\right)=h \otimes 1_{B}, \quad P\left(e \otimes 1_{A}\right)=e \otimes \alpha, \quad P\left(f \otimes 1_{A}\right)=f \otimes \alpha^{-1}, \quad \alpha \in \mathcal{B} \text { invertible. }
$$

We can decompose $P(\operatorname{sl}(2) \otimes \mathcal{A})$ into the weight spaces under the action of $h \otimes 1_{B}=$ $P\left(h \otimes 1_{A}\right)$. The weight spaces $h \otimes \mathcal{B}, e \otimes \mathcal{B}$, and $f \otimes \mathcal{B}$ correspond to the weights 0,2 , and -2 respectively. As

$$
\left[h \otimes 1_{B}, P(x \otimes a)\right]=\left[P\left(h \otimes 1_{A}\right), P(x \otimes a)\right]=P\left(\left[h \otimes 1_{A}, x \otimes a\right]\right)=P([h, x] \otimes a),
$$

for $a \in \mathcal{A}$, we obtain for $x \in\{h, e, f\}$

$$
P(h \otimes a)=h \otimes Q^{h}(a), \quad P(e \otimes a)=e \otimes Q^{e}(a), \quad P(f \otimes a)=f \otimes Q^{f}(a),
$$

with linear maps $Q^{h}, Q^{e}, Q^{f}: \mathcal{A} \rightarrow \mathcal{B}$. We will show that the map $Q^{h}$ is an algebra isomorphism. Let $a_{1}, a_{2} \in \mathcal{A}$. From $\left[e \otimes a_{1}, f \otimes a_{2}\right]=[e, f] \otimes\left(a_{1} a_{2}\right)=h \otimes\left(a_{1} a_{2}\right)$ one obtains after applying $P$ that $Q^{h}\left(a_{1} a_{2}\right)=Q^{e}\left(a_{1}\right) Q^{f}\left(a_{2}\right)$. Next we consider $\left[h \otimes a, e \otimes 1_{A}\right]=$ $[h, e] \otimes a=2 e \otimes a$. As $\alpha=Q^{e}(1)$, we obtain after applying $P$ that $Q^{e}(a)=Q^{h}(a) \cdot \alpha$. In the same way we get $Q^{f}(a)=Q^{h}(a) \cdot \alpha^{-1}$. Hence, $Q^{h}\left(a_{1} \cdot a_{2}\right)=Q^{h}\left(a_{1}\right) \cdot Q^{h}\left(a_{2}\right)$ and $Q^{h}(1)=1$. This implies that $Q^{h}$ is indeed an isomorphism of associative algebras $\mathcal{A} \rightarrow \mathcal{B}$.

The proof shows that Proposition 4.7 is also true for the case that $\mathfrak{g}$ is a reductive but non-abelian Lie algebra. In fact, it is only required that $\mathfrak{g}$ admits a Lie subalgebra isomorphic to $\operatorname{sl}(2)$.

Theorem 4.8. Let $\mathfrak{g}$ be a finite-dimensional simple Lie algebra. Then the classical current algebra $\overline{\mathfrak{g}}=\mathfrak{g} \otimes \mathbb{C}\left[z^{-1}, z\right]$ is formally rigid, but neither geometrically nor analytically rigid. Examples of nontrivial geometric deformations over the affine line $\mathbb{C}$, resp. the algebra 
$\mathbb{C}[e]$, are given by the current algebras $\overline{\mathcal{G}}_{(e)}^{s}$ of Krichever-Novikov type 44.18), 4.19) for every $s \in \mathbb{C} \cup\{\infty\}$.

Proof. First note that as $\mathfrak{g}$ is simple, the classical current algebra $\overline{\mathfrak{g}}$ is rigid by a result of Lecomte and Roger [20]. But obviously for every $s, \overline{\mathcal{G}}_{(e)}^{s}$ defines a geometric deformation of $\overline{\mathfrak{g}}$ over $\mathbb{C}[e]$. In particular we get $\overline{\mathcal{G}}_{(0)}^{s}=\overline{\mathfrak{g}}$. As by Proposition 4.6 the function algebras are not isomorphic, Proposition 4.7 implies that the algebra $\overline{\mathcal{G}}_{(e)}^{s}$ is also not isomorphic to $\overline{\mathfrak{g}}$ as long $e \neq 0$. Hence, restricted to every (algebraic-geometrically or even analytically) open neighbourhood of $e=0$, the family will not be equivalent to the trivial family. By Definition 2.5] and 2.6, $\overline{\mathfrak{g}}$ is neither geometrically, nor analytically rigid.

\subsection{Families of the centrally extended algebras.}

The above family of current algebras $\overline{\mathcal{G}}_{\left(e_{1}, e_{2}\right)}$ resp. the one-dimensional families $\overline{\mathcal{G}}_{(e)}^{s}$, can be centrally extended to a family of algebras $\widehat{\mathcal{G}}_{\left.\left(e_{1}, e_{2}\right)\right)}$, resp. $\widehat{\mathcal{G}}_{(e)}^{s}$, i. e. to families of higher genus affine Lie algebras. This can be achieved by using the defining equation (3.8).

Theorem 4.9. Assume we have a finite-dimensional Lie algebra $\overline{\mathfrak{g}}$ and a symmetric invariant bilinear form $\beta$ on $\mathfrak{g}$. For the family $\overline{\mathcal{G}}_{\left(e_{1}, e_{2}\right)}$ of current algebras 4.17) containing the classical current algebra $\overline{\mathfrak{g}}$ as special element a family of almost-graded central extensions $\widehat{\mathcal{G}}_{\left(e_{1}, e_{2}\right)}$, i.e. a family of higher genus affine Lie algebras taining the classical affine Lie algebra $\widehat{\mathfrak{g}}$ (with respect to the form $\beta$ ) as special element, is given via the geometric two-cocycle (3.8). It calculates as

$$
\gamma\left(x \otimes A_{n}, y \otimes A_{m}\right)=p\left(e_{1}, e_{2}\right) \cdot \beta(x, y) \cdot \frac{1}{2 \pi \mathrm{i}} \int_{C_{S}} A_{n} d A_{m}
$$

with

$$
\frac{1}{2 \pi \mathrm{i}} \int_{C_{S}} A_{n} d A_{m}=\left\{\begin{array}{cl}
-n \delta_{m}^{-n}, & n, m \text { even, } \\
0, & n, m \text { different parity } \\
-n \delta_{m}^{-n}+3 e_{1}(-n+1) \delta_{m}^{-n+2}+ & \\
+\left(e_{1}-e_{2}\right)\left(2 e_{1}+e_{2}\right)(-n+2) \delta_{m}^{-n+4}, & n, m \text { odd. }
\end{array}\right.
$$

Here $p\left(e_{1}, e_{2}\right)$ is an arbitrary polynomial in the variables $e_{1}$ and $e_{2}$.

The proof involves residue calculus (see Equation (3.9)) and will be postponed to an appendix. As the cocycle values (4.29) vanishes if $0 \leq n+m \leq 4$, the centrally extended algebras are almost-graded by setting $\operatorname{deg} t:=1$ (or any other fixed value).

Clearly, for $e_{1}=e_{2}=0$ we obtain the classical affine algebra $\widehat{\mathfrak{g}}$. By restricting this two-dimensional family to the lines $D_{s}$ we get one-dimensional families. For $s \neq \infty$ this amounts to replacing in the last term $\left(e_{1}-e_{2}\right)\left(2 e_{1}+e_{2}\right)$ by $e_{1}^{2}(1-s)(2+s)$. In particular, over $D_{1}$ and $D_{-2}$ it will vanish.

By the uniqueness result for almost-graded central extensions (Theorem 3.4) in the case that $\mathfrak{g}$ is simple we obtain

Corollary 4.10. If $\mathfrak{g}$ is a finite-dimensional simple Lie algebra then $\beta$ is necessarily the Cartan-Killing form, and $\widehat{\mathcal{G}}_{\left(e_{1}, e_{2}\right)}$ 4.28) is up to equivalence the unique almost graded central extension of the family of current algebras $\overline{\mathcal{G}}_{\left(e_{1}, e_{2}\right)}$. 
Based on the classification results of $[34$ in the semi-simple case, Corollary 4.10 remains true if one replaces the factor $p\left(e_{1}, e_{2}\right) \beta(x, y)$ by $\sum_{i=1}^{K} p_{i}\left(e_{1}, e_{2}\right) \beta_{i}(x, y)$, where again $p_{i}$ are polynomials, and $\beta_{i}$ are the Cartan-Killing forms of the $K$ simple summands of $\overline{\mathfrak{g}}$. A similar statement is true for the reductive case, now additionally requiring the defining cocycle to be $\mathcal{L}$-invariant (see 34 for the definition).

\section{Geometric DeGenerations}

It might be quite instructive to identify geometrically all the algebras corresponding to the singular cubic situations. Above, by comparing the structure constants we identified $\overline{\mathcal{G}}_{(0,0)}$ and $\overline{\mathcal{G}}^{(s)}$ for $s=1$ or -2 with algebras which are related to the genus zero situation. The geometric scheme behind this is that in each case the desingularisation (or normalization) of the singular cubic is the projective line. By pulling back functions on the singular cubics we obtain functions on the desingularisation. But not necessarily all functions on the desingularisation will be obtained as pullbacks. One also has to keep track of the poles. In 31 the situation is analysed in complete details.

Three different situations appear.

(I) All three $e_{1}, e_{2}$ and $e_{3}$ coincide. The normalization $e_{1}+e_{2}+e_{3}=0$ implies necessarily that they are zero. We obtain the cuspidal cubic where the singular point is also a point of possible poles. Over the singular point there is only one point. Hence we obtain an identification of the algebra $\mathcal{A}_{(0,0)}$ with the full algebra

$\mathbb{C}\left[z, z^{-1}\right]$, and furthermore the identification of the current algebra $\overline{\mathcal{G}}_{(0,0)}$ with the classical current algebra $\overline{\mathfrak{g}}$.

(II) If two of the $e_{i}$ coincide (but not all three), we obtain the nodal cubic. Over the singular point we have two points on the desingularisation. Let the desingularisation be chosen such that the points $\alpha$ and $-\alpha$ lie above the singular point. We have to distinguish two sub-cases.

(IIa) Either $e_{1}=e_{2} \neq e_{3}$ or $e_{1}=e_{3} \neq e_{2}$, then the singular point (the node) will become a possible pole. This situation occurs if we approach points from $D_{1}^{*} \cup D_{-2}^{*}$. The algebra generated by the pullbacks will be the full function algebra of the 3-point algebra $\mathcal{V}_{(\alpha)}$. Hence, we see geometrically the already remarked isomorphy. On the level of the current algebra we get the identification of $\mathcal{Y}_{(\alpha)}$ with $\overline{\mathcal{G}}_{(e)}^{1}$ and $\overline{\mathcal{G}}_{(e)}^{-2}$.

(IIb) If $e_{1} \neq e_{2}=e_{3}$, then the point of a possible pole will remain non-singular. This appears if we approach a point of $D_{-1 / 2}^{*}$. For the pullbacks of the functions it is now necessary that they have the same value at the points $\alpha$ and $-\alpha$. Hence, all elements of the algebra generated by the pullbacks will have the same property. We describe this algebra in the following.

Proposition 5.1. The set of elements

$$
A_{n}:= \begin{cases}z^{n}, & n \text { even }, \\ z^{n}-\alpha^{2} z^{n-2}=z^{n-2}\left(z^{2}-\alpha^{2}\right), & n \text { odd },\end{cases}
$$

for $n \in \mathbb{Z}$ form a basis of the subalgebra $\mathcal{W}_{(\alpha)}$ of meromorphic functions on $\mathbb{P}^{1}$ which are holomorphic outside 0 and $\infty$ and have the same value at $\alpha$ and $-\alpha$. The algebra structure 
is given by

$$
A_{n} \cdot A_{m}=\left\{\begin{array}{cc}
A_{n+m}, & \text { for } n \text { or } m \text { even }, \\
A_{n+m}-2 \alpha^{2} A_{n+m-2} & \\
+\alpha^{4} A_{n+m-4}, & \text { for } n \text { and } m \text { both odd } .
\end{array}\right.
$$

For this subalgebra we have $\mathcal{W}_{(\alpha)} \cong \mathcal{A}^{-1 / 2}$ and it is not isomorphic to $\mathbb{C}\left[z^{-1}, z\right]$.

Proof. Obviously these elements lie in this subalgebra and form a basis of the subalgebra of sums of all even functions (without any restriction) and odd functions which vanish at $\pm \alpha$. Let $f$ be a function fulfilling the conditions for being a member of $\mathcal{W}_{(\alpha)}$. Decompose it into its symmetric and antisymmetric part

$$
f=f_{1}+f_{2}, \quad f_{1}(x)=1 / 2(f(x)+f(-x)), \quad f_{2}(x)=1 / 2(f(x)-f(-x)) .
$$

Obviously $f_{1}$ also fulfills the conditions, hence $f_{2}$ too and we get $f_{2}(-\alpha)=f_{2}(\alpha)$. Being an antisymmetric function this implies that $f_{2}$ has to vanish at $\pm \alpha$, and $f$ is a linear combination of the elements $A_{n}$. By setting $\alpha=\mathrm{i} \sqrt{\frac{3 e_{1}}{2}}$ we immediately see that $\mathcal{W}_{(\alpha)} \cong$ $\mathcal{A}^{-1 / 2}$. Hence by Proposition 4.6. $\mathcal{W}_{(\alpha)}$ is not isomorphic to $\mathbb{C}\left[z^{-1}, z\right]$.

Clearly again the algebras $\mathcal{W}_{(\alpha)}$ are isomorphic for different $\alpha \neq 0$.

The above mentioned identifications extend to the current algebras and we obtain another one-dimensional algebraic-geometric deformation family $\mathcal{Z}_{(\alpha)}$ of the current algebra.

Proposition 5.2. In the two-parameter family [4.17), the current algebras for the singular cubic cases are isomorphic as follows:

$$
\begin{aligned}
\overline{\mathcal{G}}_{(0,0)} & \cong \overline{\mathfrak{g}}=\mathfrak{g} \otimes \mathbb{C}\left[z, z^{-1}\right], \\
\overline{\mathcal{G}}^{1} \cong \overline{\mathcal{G}}^{-2} & \cong \mathfrak{g} \otimes \mathcal{V}, \\
\overline{\mathcal{G}}^{-1 / 2} & \cong \mathfrak{g} \otimes \mathcal{Z} .
\end{aligned}
$$

Recall that here we denoted the family with the same letter as the isomorphy type of the generic member.

These considerations extend to the centrally extended families of algebras introduced in Section 4.5, see Proposition A.1.

\section{Cohomology Classes of the Deformations}

Let $\overline{\mathcal{G}}_{t}$ be a one-parameter deformation of the current algebra $\overline{\mathfrak{g}}$ with Lie structure

$$
[\alpha, \beta]_{t}=[\alpha, \beta]+t^{k} \omega_{0}(\alpha, \beta)+t^{k+1} \omega_{1}(\alpha, \beta)+\cdots,
$$

such that $\omega=\omega_{0}$ is non-vanishing. As explained in Section 2.1. the bilinear map $\omega$ will be an element of $\mathrm{C}^{2}(\overline{\mathfrak{g}}, \overline{\mathfrak{g}})$.

For the global families $\overline{\mathcal{G}}_{(e)}^{s}$ over the affine line $D_{s}$ appearing in Section 4 we obtain as first nontrivial contribution the following two-cocycles $(s \neq \infty)$ :

$$
\omega\left(x \otimes A_{n}, y \otimes A_{m}\right)= \begin{cases}0, & \text { for } n \text { or } m \text { even, } \\ {[x, y] \otimes A_{n+m-2}} & \text { for } n \text { and } m \text { both odd. }\end{cases}
$$


For $D_{\infty}$ we get

$$
\omega\left(x \otimes A_{n}, y \otimes A_{m}\right)= \begin{cases}0, & \text { for } n \text { or } m \text { even, } \\ {[x, y] \otimes A_{n+m-4},} & \text { for } n \text { and } m \text { both odd. }\end{cases}
$$

Here we used the symbols $x \otimes A_{n}$ for the basis elements $x \otimes z^{n}$ also in the classical case. It should be pointed out that the families corresponding to the singular cubic situations are included for $s=1,-2$ or $s=-1 / 2$.

Proposition 6.1. Let $\mathfrak{g}$ be an arbitrary finite dimensional Lie algebra. The two-cocycles (6.2) and (6.3) for the algebra $\overline{\mathfrak{g}}$ are cohomologically trivial. Hence, considered as infinitesimal deformations of $\overline{\mathfrak{g}}$, the families $\overline{\mathcal{G}}_{(e)}^{\text {are trivial. }}$

Proof. We verify directly that $\omega=d_{1} \eta$ for $\eta: \overline{\mathfrak{g}} \rightarrow \overline{\mathfrak{g}}$ defined by

$$
\eta\left(x \otimes A_{n}\right)=\left\{\begin{array} { l l } 
{ 0 , } & { n \text { even, } } \\
{ - \frac { 1 } { 2 } x \otimes A _ { n - 2 } , } & { n \text { odd, } }
\end{array} \quad \text { resp. } \quad \left\{\begin{array}{ll}
0, & n \text { even }, \\
-\frac{1}{2} x \otimes A_{n-4}, & n \text { odd } .
\end{array}\right.\right.
$$

Clearly, if $\mathfrak{g}$ is a simple Lie algebra the vanishing of the cohomology class follows from the formal rigidity of $\overline{\mathfrak{g}}(20])$.

Remark. As an intermediate step we constructed (inside the category of commutative and associative algebras) nontrivial algebraic-geometric deformations $\mathcal{A}_{(e)}^{s}$ of the algebra of Laurent polynomials $\mathbb{C}\left[z^{-1}, z\right]$. One sees here the same effect as in the Lie case. The relevant cohomology theory is the Harrisson cohomology (see Section [2.8). As $\mathbb{C}\left[z^{-1}, z\right]$ is the algebra of regular functions on the smooth affine curve $\mathbb{P}^{1} \backslash\{0, \infty\}$, it is a smooth affine algebra and its Harrisson two-cohomology vanishes [15, Thm.22]. Again we get that, despite the fact that this algebra is infinitesimally and formally rigid, there exist nontrivial local geometric families. See Kontsevich 17 for his concept of semi-formal deformations (related to filtrations of certain type) to overcome this discrepancy. Indeed, by the almost-gradedness of the involved families considered in this article, the families $\mathcal{A}_{(e)}^{s}$ are semi-formal deformations in his sense. Further considerations in these directions have to be postponed to another article.

As the Harrison two-cohomology vanishes, the defining cocycle for these families will be a coboundary. One verifies immediately that it is the coboundary of the linear form

$$
\eta\left(A_{n}\right)=\left\{\begin{array} { l l } 
{ 0 , } & { n \text { even, } } \\
{ \frac { 1 } { 2 } A _ { n - 2 } , } & { n \text { odd, } }
\end{array} \quad \text { resp. } \quad \left\{\begin{array}{ll}
0, & n \text { even, } \\
\frac{1}{2} A_{n-4}, & n \text { odd } .
\end{array}\right.\right.
$$

\section{Appendix A. Proof of Theorem 4.9}

In this appendix we will show that the geometrically defined central extension (4.17) of the family $\overline{\mathcal{G}}_{\left(e_{1}, e_{2}\right)}$ has the form (4.28) if expressed for pairs of generators $x \otimes A_{n}$, $x \in \mathfrak{g}$ and $A_{n}, n \in \mathbb{Z}$ the (homogeneous) basis elements of $\mathcal{A}_{\left(e_{1}, e_{2}\right)}$. It is enough to show the expression (4.29) for $\frac{1}{2 \pi \mathrm{i}} \int_{C_{S}} A_{n} d A_{m}$ for every pair of basis elements of the function algebra $\mathcal{A}_{\left(e_{1}, e_{2}\right)}$. As a remark aside, this integral defines central extensions of the algebra of functions considered as abelian Lie algebras, see 33 .

As the integration is over a separating cycle $C_{S}$, the integral can be calculated by calculating residues at either one of the possible poles, $\left(e_{1}, 0\right)$ or $\infty$. From the point $\infty$ the 
residue has to be taken with a minus sign. One possible way to calculate the residue is to change to the complex-analytic picture. This means we use the fact that $X$ corresponds to the elliptic Weierstraß $\wp$-function and $Y$ to its derivative $\wp^{\prime}$. They are doubly-periodic functions on the complex plane with respect to the lattice $\Gamma=\mathbb{Z} \oplus \tau \mathbb{Z}$ with $\tau \in \mathbb{C}$, im $\tau>0$. We use the variable $z$ for the complex variable in the plane. With this identification the complex one-dimensional torus $\mathbb{C} / \Gamma$ is analytically isomorphic to the (projective) elliptic curve. The variable $\tau$ fixes the isomorphy class of the torus, the parameters $e_{1}, e_{2}, e_{3}$ the isomorphy class of the elliptic curve. Clearly, they are related. One relation which is important for us is

$$
\wp\left(\frac{1}{2}\right)=e_{1}, \quad \wp\left(\frac{\tau}{2}\right)=e_{2}, \quad \wp\left(\frac{\tau+1}{2}\right)=e_{3} .
$$

Note also that the functions $\wp$ and $\wp^{\prime}$ depend on $\tau$ and hence also on the parameters $e_{1}, e_{2}$ and $e_{3}$.

We recall the following well-know facts from the theory of elliptic functions. The function $\wp$ fulfills the differential equation

$$
\left(\wp^{\prime}\right)^{2}=4\left(\wp-e_{1}\right)\left(\wp-e_{2}\right)\left(\wp-e_{3}\right)=4 \wp^{3}-g_{2} \wp-g_{3} .
$$

We have

$$
e_{1}+e_{2}+e_{3}=0, \quad g_{2}=-4\left(e_{1} e_{2}+e_{1} e_{3}+e_{2} e_{3}\right), \quad g_{3}=4\left(e_{1} e_{2} e_{3}\right) .
$$

The function $\wp$ is an even meromorphic function with poles of order two at the points of the lattice and holomorphic elsewhere. The function $\wp^{\prime}$ is an odd meromorphic function with poles of order three at the points of the lattice and holomorphic elsewhere. It has zeros of order one at the points $1 / 2, \tau / 2$ and $(1+\tau) / 2$ and all its translates under the lattice. The zeros are of order one.

For the Laurent series expansion at $z=0$ we obtain

$$
\wp(z)=\frac{1}{z^{2}}\left(1+\frac{g_{2}}{20} z^{4}+O\left(z^{6}\right)\right), \quad \wp(z)-e_{1}=\frac{1}{z^{2}}\left(1+e_{1} z^{2}+\frac{g_{2}}{20} z^{4}+O\left(z^{6}\right)\right) .
$$

In terms of these functions the basis elements $A_{n}$ can be expressed as

$$
A_{2 k}=\left(\wp-e_{1}\right)^{k}, \quad A_{2 k+1}=\frac{1}{2} \wp^{\prime} \cdot\left(\wp-e_{1}\right)^{k-1} \quad k \in \mathbb{Z} .
$$

Note that $\left(\wp-e_{1}\right)$ has a pole of order two at $z=0$ and a zero of order two at $z=1 / 2$. It has a Laurent expansion in even powers of $z$. Hence the same is true for $A_{2 k}$. Furthermore, $A_{2 k+1}(z)=\frac{1}{2 k} \frac{d}{d z} A_{2 k}(z)$. This allows us to determine the Laurent expansion of $A_{2 k+1}$ easily. Its Laurent expansion consists of only odd powers of $z$.

Our first conclusion is that the differential $A_{2 k+1} A_{2 l}^{\prime} d z$ has only even terms in its Laurent expansion. Hence, as the residue is the coefficient of $\frac{1}{z}$, there is no residue, and the cocycle values evaluated for pairs of basis elements of different parity is zero, as claimed (4.29).

Due to the residue theorem (on the torus) the total residue of any differential has to vanish. Hence, there will be only a residue at one point if the differential has poles at both points, $z=0$ and $z=1 / 2$. In the even case, $A_{2 k} A_{2 l}^{\prime} d z$, the pole order at 0 is $2(k+l)+1$, and at $1 / 2$ it is $(-2 k)+(-2 l+1)=-2(k+l)+1$. Hence, a possibly nonvanishing value could appear only if $l=-k$ (or, equivalently $m=-n$ ). In the odd case, $A_{2 k+1} A_{2 l+1}^{\prime} d z$, we have poles at 0 of order $(2 k+1)+(2 l+2)=2(k+l)+3$, and at $1 / 2$ of 
order $(-2 k+1)+(-2 l+2)=-2(k+l)+3$. Hence, there could only be a contribution if $l=-k-1,-k$, or $-k+1$ corresponding to $m=-n,-n+2$, or $-n+4$.

To calculate the residue we have to consider the Laurent expansion of the generators and their derivatives. Starting from (A.4) we get

$$
\begin{aligned}
& A_{2 k}=\frac{1}{z^{2 k}}\left(1+k e_{1} z^{2}+k\left(\frac{k-1}{2} e_{1}^{2}+\frac{g_{2}}{20}\right) z^{4}+O\left(z^{6}\right),\right) \\
& A_{2 k}^{\prime}= \frac{1}{z^{2 k+1}}\left(-2 k+O\left(z^{2}\right)\right), \\
& A_{2 k+1}=\frac{1}{2 k} A_{2 k}^{\prime}= \frac{1}{z^{2 k+1}}\left(-1+(-k+1) e_{1} z^{2}+(-k+2)\left(\frac{k-1}{2} e_{1}^{2}+\frac{g_{2}}{20}\right) z^{4}+O\left(z^{6}\right),\right) \\
& A_{2 k+1}^{\prime}= \frac{1}{z^{2 k+2}}\left(2 k+1+(-k+1)(-2 k+1) e_{1} z^{2}+\right. \\
&\left.+(-k+2)(-2 k+3)\left(\frac{k-1}{2} e_{1}^{2}+\frac{g_{2}}{20}\right) z^{4}+O\left(z^{6}\right),\right)
\end{aligned}
$$

For the even pairing, i.e. $n=2 k, m=2 l$, we obtain in case $l=-k$

$$
A_{2 k} A_{-2 k}^{\prime} d z=\frac{1}{z}\left(2 k+O\left(z^{2}\right)\right) \text {. }
$$

Hence the residue at $z=0$ is $2 k=m$, and as it has to be taken negatively, we obtain $\frac{1}{2 \pi \mathrm{i}} \int_{C_{S}} A_{n} d A_{-n}=-n$, as claimed in (4.29).

For the odd pairing, i.e. $n=2 k+1, m=2 l+1$, we have to multiply the Laurent expansions of $A_{2 k+1}$ and $A_{2 l+1}^{\prime}$. The resulting Laurent series is

$$
\frac{1}{z^{2(k+l)+3}}\left(a(k, l)+b(k, l) z^{2}+c(k, l) z^{4}+O\left(z^{6}\right)\right) d z
$$

with $a(k, l), b(k, l)$ and $c(k, l)$ obtained by collecting the corresponding contributions to these orders. In particular, we obtain

$$
a(k, l)=-(2 l+1), \quad b(k, l)=e_{1}((-k+1)(2 l+1)+l-1)
$$

Now the residue at $z=0$ can be easily calculated.

For $l=-k-1$ we obtain as residue $a(k,-k-1)=(2 k+1)=n$.

For $l=-k$ the residue is $b(k,-k)=-3 e_{1}(-n+2)$.

For $l=-k+1$ the residue is

$$
c(k,-k+1)=-\left(e_{1}-e_{2}\right)\left(2 e_{1}+e_{2}\right)(-n+4) .
$$

For the last calculation we have to express $g_{2}$ by the $e_{i}$ 's as given in (A.3) and replace $e_{3}=-\left(e_{1}+e_{2}\right)$. Finally, it should not be forgotten to change the sign to obtain the cocycle values claimed in (4.28).

Strictly speaking, this derivation via the elliptic functions is valid above the points outside of the collection of lines $D_{1} \cup D_{-2} \cup D_{-1 / 2}$. But being a cocycle is a closed condition, hence the bilinear form will also be a cocycle for the algebras not corresponding to elliptic algebras. It will be obtained by specializing the values of $e_{1}$ and $e_{2}$. 
Nevertheless, it is an easy exercise to calculate the cocycle for the genus zero algebras using again residues. In this case the functions are rational functions, hence the calculations will be easier. For a convenient reference, let us note here the form of the cocycle for the singular situation.

Proposition A.1. (a) For the current algebra $\overline{\mathcal{G}}_{(0,0)}=\overline{\mathfrak{g}}$ the centrally extended algebra is given by the cocycle $(p \in \mathbb{C})$

$$
\gamma\left(x \otimes A_{n}, y \otimes A_{m}\right)=p \cdot \beta(x, y) \cdot(-n) \delta_{m}^{-n} .
$$

(b) For the three-point genus zero family of current algebras $\mathcal{Y}_{(\alpha)}$ (4.22) the family of centrally extended algebras is given by the cocycle

$$
\gamma\left(x \otimes A_{n}, y \otimes A_{m}\right)=p(\alpha) \cdot \beta(x, y) \cdot \frac{1}{2 \pi \mathrm{i}} \int_{C_{S}} A_{n} d A_{m},
$$

with a polynomial $p$ in $\alpha$ and

$$
\frac{1}{2 \pi \mathrm{i}} \int_{C_{S}} A_{n} d A_{m}=\left\{\begin{array}{cl}
-n \delta_{m}^{-n}, & n, m \text { even } \\
0, & n, m \text { different parity } \\
-n \delta_{m}^{-n}+\alpha^{2}(-n+1) \delta_{m}^{-n+2}, & n, m \text { odd } .
\end{array}\right.
$$

(c) For the family of current algebras $\mathcal{Z}_{(\alpha)}$ defined in Section 5 the family of centrally extended algebras is given by the cocycle

$$
\gamma\left(x \otimes A_{n}, y \otimes A_{m}\right)=p(\alpha) \cdot \beta(x, y) \cdot \frac{1}{2 \pi \mathrm{i}} \int_{C_{S}} A_{n} d A_{m},
$$

with a polynomial $p$ in $\alpha$ and

$$
\frac{1}{2 \pi \mathrm{i}} \int_{C_{S}} A_{n} d A_{m}=\left\{\begin{array}{cl}
-n \delta_{m}^{-n}, & n, m \text { even } \\
0, & n, m \text { different parity } \\
-n \delta_{m}^{-n}-2 \alpha^{2}(-n+1) \delta_{m}^{-n+2}+ & \\
+\alpha^{4}(-n+2) \delta_{m}^{-n+4}, & n, m \text { odd } .
\end{array}\right.
$$

Remark. The bilinear form $\gamma(f, g)=\frac{1}{2 \pi \mathrm{i}} \int_{C_{S}} f d g$ on the function algebra $\mathcal{A}=\mathcal{A}_{\left(e_{1}, e_{2}\right)}$ appearing in the definition of the cocycle for the current algebra is a cocycle for $\mathcal{A}$ (considered as abelian Lie algebra) defining a central extension of it, i.e. an infinite dimensional Heisenberg algebra. In 33] it was shown that this cocycle is up to multiplication with a constant the unique local and multiplicative cocycle for $\mathcal{A}$. Note that because $\mathcal{A}$ is abelian, there are no non-trivial coboundaries. A cocycle is called local if there are constants $M$ and $N$ such that

$$
\gamma\left(\mathcal{A}_{n}, \mathcal{A}_{m}\right) \neq 0, \quad \text { implies } \quad M \leq n+m \leq L .
$$

Being a local cocycle says, that the central extension defined via the cocycle is almostgraded. A cocycle is called multiplicative if

$$
\gamma(f g, h)+\gamma(g h, f)+\gamma(h f, g)=0, \quad f, g, h \in \mathcal{A} .
$$

In the quoted article the uniqueness was shown by applying a recursive procedure using the locality and the multiplicativity of the cocycle and the almost-graded structure of the 
algebra $\mathcal{A}$. From this it is clear that the total cocycle $\frac{1}{2 \pi \mathrm{i}} \int_{C_{S}} f d g$ is already fixed by one non-vanishing cocycle value (let's say $\gamma\left(A_{2}, A_{-2}\right)$ ) and the structure constants (4.15) of the algebra. Instead going through the Laurent series calculations above, one could equally well go through the combinatorics of the recursion in 33] using the structure of $\mathcal{A}$. We will supply an alternative proof in the next appendix. From this proof the similarity of the structure equations (4.15) with the formulas for the central extensions (4.29) will become clear.

\section{Appendix B. A Different proof of Theorem 4.9}

First note that as antisymmetric and bilinear form, $\gamma(f, g)=\frac{1}{2 \pi \mathrm{i}} \int_{C_{S}} f d g$ defines a twococycle for the abelian Lie algebra $\mathcal{A}$. Furthermore, note that there does not exist any non-trivial coboundary.

Recall that we are working over a compact Riemann surface, resp. a smooth projective curve over $\mathbb{C}$. By the residue theorem the total residue of every differential has to vanish. Hence, a differential having a pole with a (point-)residue has to have at least another pole. This implies that with respect to the grading introduced, the cocycle is local (see (A.16)). This can be seen by estimating the pole orders of the differential $A_{n} d A_{m}$ at the points 0 and $1 / 2$.

As the (point-)residue of an exact differential of a meromorphic function is zero we obtain

$$
0=\frac{1}{2 \pi \mathrm{i}} \int_{C_{S}} d(f g h)=\frac{1}{2 \pi \mathrm{i}} \int_{C_{S}} f g d(h)+\frac{1}{2 \pi \mathrm{i}} \int_{C_{S}} g h d(f)+\frac{1}{2 \pi \mathrm{i}} \int_{C_{S}} h f d(g) .
$$

This condition is exactly the multiplicativity of the cocycle $\gamma$.

Recall the structure of the algebra:

$$
A_{n} \cdot A_{m}= \begin{cases}A_{n+m}, & n \text { or } m \text { even, } \\ A_{n+m}+a A_{n+m-2}+b A_{n+m-4}, & n \text { and } m \text { odd }\end{cases}
$$

with $a=3 e_{1}$ and $b=\left(e_{1}-e_{2}\right)\left(2 e_{1}+e_{2}\right)$.

For the set of values $\gamma\left(A_{n}, A_{m}\right)$ we introduce its level $l=n+m$. In the following we will use induction on the level $l$.

1. If $l<0$ then $\gamma\left(A_{n}, A_{m}\right)=\gamma\left(A_{n}, A_{-n+l}\right)=0$. This follows immediately from the fact that $\gamma$ is a local cocycle (see [33], where the inverted grading was used). Alternatively, it can be easily checked by showing that there will be no pole at $z=0$ as long as $l<0$.

2. We have to calculate one cocycle value for normalization.

$$
\begin{aligned}
\gamma\left(A_{2}, A_{-2}\right)=-\gamma\left(A_{-2}, A_{2}\right)=\operatorname{res}_{z=0}((\wp(z) & \left.\left.-e_{1}\right)^{-1} \frac{d}{d z}\left(\wp(z)-e_{1}\right)\right) \\
& =\operatorname{res}_{z=0}\left(\left(\wp(z)-e_{1}\right)^{-1} \wp^{\prime}\right)=-2 .
\end{aligned}
$$

3.

Lemma B.1. $\gamma\left(A_{n}, A_{0}\right)=0$ for all $n \in \mathbb{Z}$. 
Proof.

$\gamma\left(A_{n}, A_{0}\right)=\gamma\left(A_{n}, A_{0} \cdot A_{0}\right)=-\gamma\left(A_{n} \cdot A_{0}, A_{0}\right)-\gamma\left(A_{0} \cdot A_{0}, A_{n}\right)=-\gamma\left(A_{n}, A_{0}\right)+\gamma\left(A_{n}, A_{0}\right)=0$.

Above we used the multiplicativity. Again with the multiplicativity we obtain

Lemma B.2. $\gamma(f \cdot f, f)=0$.

4. Let $l=0$, and $n$ of arbitrary parity. We calculate

$$
\begin{array}{r}
\gamma\left(A_{n+1}, A_{-(n+1)}\right)=\gamma\left(A_{n} A_{1}, A_{-(n+1)}\right)=-\gamma\left(A_{1} A_{-(n+1)}, A_{n}\right)-\gamma\left(A_{-(n+1)} A_{n}, A_{1}\right) \\
=-\gamma\left(A_{-n}, A_{n}\right)-\gamma\left(A_{-1}, A_{1}\right)=\gamma\left(A_{n}, A_{-n}\right)+\gamma\left(A_{1}, A_{-1}\right) .
\end{array}
$$

Here certain remarks are necessary, as this chain of equalities is not that innocent as it looks. For the first equality note that if $n$ is even we can indeed replace $A_{n+1}$ by $A_{n} A_{1}$, using (B.2). If $n$ is odd then $A_{n+1}=A_{n} A_{1}-a A_{n-1}-b A_{n-3}$. But we have $\gamma\left(A_{n-1}, A_{-(n+1)}\right)=\gamma\left(A_{n-3}, A_{-(n+1)}\right)=0$, as their levels are -2 , resp. -4 , and for those levels the cocycle is vanishing. Hence, indeed the first inequality is true for every parity. Using the multiplicativity, we replace the one cocycle by the negative of its two partner cocycles. Now we use the same kind of arguments as above to replace the products for them.

Altogether we obtain a simple recursion relation which has as unique solution

$$
\gamma\left(A_{n}, A_{-n}\right)=n \cdot \gamma\left(A_{1}, A_{-1}\right)=(-n) .
$$

The last equality follows from the cocycle value we calculated in Step 2.

5. We consider $(n,-n+l)$ with $l \neq 0$, such that either both entries are even (this says $l$ is even), or $n$ is odd and the other entry is even (this says $l$ is odd). We claim that all cocycle values for such pairs vanish. For $l$ even, we will show the claim directly. For $l$ odd we will use induction. First note that for $l$ negative the values will be zero by locality. Hence the start of the induction is in the odd case trivially true. We calculate

$$
\begin{array}{r}
\gamma\left(A_{n}, A_{-n+l}\right)=\gamma\left(A_{n-2} A_{2}, A_{-n+l}\right)=-\gamma\left(A_{2} A_{-n+l}, A_{n-2}\right)-\gamma\left(A_{-n+l} A_{n-2}, A_{2}\right) \\
=\gamma\left(A_{n-2}, A_{-(n-2)+l}\right)+\gamma\left(A_{2}, A_{l-2}\right) .
\end{array}
$$

Lemma B.3. For $l \neq 0$ we have $\gamma\left(A_{2}, A_{l-2}\right)=0$.

Proof. By the locality $\gamma\left(A_{2}, A_{l-2}\right)=0$ if $l \leq 0$. First we have to consider $l=1,2,3,4$ individually.

For $l=1$ we get

$$
\begin{aligned}
\gamma\left(A_{2}, A_{-1}\right) & =\gamma\left(A_{1} A_{1}, A_{-1}\right)=-\gamma\left(A_{1} A_{-1}, A_{1}\right)-\gamma\left(A_{-1} A_{1}, A_{1}\right)= \\
& -2 \gamma\left(A_{-1} A_{1}, A_{1}\right)=-2\left(\gamma\left(A_{0}, A_{1}\right)+a \gamma\left(A_{-2}, A_{1}\right)+b \gamma\left(A_{-4}, A_{1}\right)\right)=0 .
\end{aligned}
$$

Here we used that the cocycle vanishes if the level is negative, the multiplicativity and Lemma B.1. 
For $l=2$ we have $\gamma\left(A_{2}, A_{0}\right)=0$ by Lemma B.1

For $l=3$ we calculate

$$
\gamma\left(A_{2}, A_{1}\right)=\gamma\left(A_{1} A_{1}-a A_{0}-b A_{-2}, A_{1}\right)=\gamma\left(A_{1} A_{1}, A_{1}\right)=0 .
$$

(We used also Lemma B.2.)

For $l=4$ we have $\gamma\left(A_{2}, A_{2}\right)=0$ by the antisymmetry.

Now let $l \geq 5$. We showed above that

$$
\gamma\left(A_{r}, A_{-r+l}\right)-\gamma\left(A_{r-2}, A_{-(r-2)+l}\right)+\gamma\left(A_{2}, A_{l-2}\right)=0 .
$$

If $l$ is even we let $r=4,6, \ldots, l-2$ and sum this $(l-4) / 2$ equations up. As sum we obtain

$$
\gamma\left(A_{l-2}, A_{2}\right)-\gamma\left(A_{2}, A_{l-2}\right)-\frac{l-4}{2} \gamma\left(A_{2}, A_{l-2}\right)=0 .
$$

Hence,

$$
\frac{l-4}{2} \gamma\left(A_{2}, A_{l-2}\right)=0
$$

and as $l>4, \gamma\left(A_{2}, A_{l-2}\right)=0$.

For $l$ odd we sum up the equations for $r=3,5, \ldots, l-2$. These are $(l-3) / 2$ equations. The sum calculates to

$$
\gamma\left(A_{1}, A_{l-1}\right)+\frac{l-1}{2} \gamma\left(A_{2}, A_{l-2}\right)=0 .
$$

We calculate

$$
\gamma\left(A_{2}, A_{l-2}\right)=\gamma\left(A_{1} A_{1}, A_{l-2}\right)-a \gamma\left(A_{0}, A_{l-2}\right)-b \gamma\left(A_{-2}, A_{l-2}\right)=\gamma\left(A_{1} A_{1}, A_{l-2}\right),
$$

as the other summands vanish by induction. Hence,

$$
\gamma\left(A_{2}, A_{l-2}\right)=-\gamma\left(A_{1} A_{l-2}, A_{1}\right)-\gamma\left(A_{l-2} A_{1}, A_{1}\right)=2 \gamma\left(A_{1}, A_{l-1}+a A_{l-3}+b A_{l-5}\right) .
$$

By induction what remains is

$$
\gamma\left(A_{2}, A_{l-2}\right)=2 \gamma\left(A_{1}, A_{l-1}\right) .
$$

Hence, from (B.13) it follows that also in this case $\gamma\left(A_{2}, A_{l-2}\right)=0$.

This proofs shows also

Lemma B.4. For $l$ odd we have $\gamma\left(A_{1}, A_{l-1}\right)=0$.

If $n$ is even from (B.7) it follows that $\gamma\left(A_{n}, A_{-n+l}\right)=\frac{n}{2} \gamma\left(A_{2}, A_{l-2}\right)$. Hence by LemmaB.3. all cocycle values will vanish.

If $n$ is odd, $l$ is odd and we get by Lemma B.4 and Lemma B.3 that the starting value and the increment for the recursion are zero. Hence, also here all cocycle values will vanish.

6. It remains to consider the case that both entries in $(n,-n+l)$ are odd, and $l>0$. In any case, $l$ will be an even number.

$$
\begin{array}{r}
\gamma\left(A_{n}, A_{-n+l}\right)=\gamma\left(A_{n-1} A_{1}, A_{-n+l}\right)=-\gamma\left(A_{1} A_{-n+l}, A_{n-1}\right)-\gamma\left(A_{-n+l} A_{n-1}, A_{1}\right) \\
=-\gamma\left(A_{-n+l+1}+a A_{-n+l-1}+b A_{-n+l-3}, A_{n-1}\right)-\gamma\left(A_{l-1}, A_{1}\right) .
\end{array}
$$


In the first expression there are only (even,even) combinations, which have only a nonvanishing value if they are $(k,-k)$. Altogether we obtain

$$
\gamma\left(A_{n}, A_{-n+l}\right)=-a(n-1) \delta_{l}^{2}--b(n-1) \delta_{l}^{4}+\gamma\left(A_{1}, A_{l-1}\right) .
$$

For $l=2$ we obtain $\gamma\left(A_{1}, A_{l-1}\right)=\gamma\left(A_{1}, A_{1}\right)=0$. Hence, $\gamma\left(A_{n}, A_{-n+2}\right)=a \cdot(-n+1)$. For $l=4$ we calculate

$$
\gamma\left(A_{1}, A_{3}\right)=\gamma\left(A_{1}, A_{2} A_{1}\right)=-\gamma\left(A_{1}, A_{1} A_{2}\right)-\gamma\left(A_{2}, A_{1} A_{1}\right) .
$$

This implies

$$
\gamma\left(A_{1}, A_{3}\right)=\frac{1}{2} \gamma\left(A_{1} A_{1}, A_{2}\right)=\frac{1}{2} \gamma\left(A_{2}+a A_{0}+A_{-2}, A_{2}\right)=\frac{1}{2} \gamma\left(A_{-2}, A_{2}\right)=b .
$$

Hence, $\gamma\left(A_{n}, A_{-n+4}\right)=b \cdot(-n+2)$.

For $l>4$ we have $\gamma\left(A_{n}, A_{-n+l}\right)=\gamma\left(A_{1}, A_{l-1}\right)$. This says that for level $l$ all values are equal to the same constant $\gamma\left(A_{1}, A_{l-1}\right)$. This is only possible if this constant is zero, as

$$
\gamma\left(A_{1}, A_{l-1}\right)=-\gamma\left(A_{l-1}, A_{1}\right)=-\gamma\left(A_{l-1}, A_{-(l-1)+k}\right)=-\gamma\left(A_{1}, A_{l-1}\right) .
$$

Hence, $\gamma\left(A_{n}, A_{-n+l}\right)=0$ for $l>4$. This closes the calculation of the cocycle values.

\section{REFERENCES}

[1] Belavin, A.A., Polyakov, A.M., Zamolodchikov, A.B.: Infinite conformal symmetry in two-dimensional quantum field theory. Nucl. Phys. B 241, 333-380 (1084)

[2] Feigin, B. L., Fuchs, D. B.: Cohomologies of Lie groups and Lie algebras. In: Onishchik, A.L., and Vinberg, E.B. (eds) Lie Groups and Lie Algebras II. Encyclopaedia of Math. Sciences, Vol. 21, pp. 125-215, Springer, New-York, Berlin, Heidelberg, Tokyo, 2000.

[3] Fialowski, A.: Deformations of the Lie algebra of vector fields on the line. Uspekhi Mat. Nauk, 38 201-202 (1983); English translation: Russian Math. Surveys, 38, No. 1, 185-186 (1983).

[4] Fialowski, A.: Deformations of nilpotent Kac-Moody algebras, Stud. Sci. Math. Hung. $19465-483$ (1984).

[5] Fialowski, A.: Deformations of Lie algebras. Math. USSR Sbornik 55, 467-472 (1986).

[6] Fialowski, A.: An example of formal deformations of Lie algebras. In: Proceedings of NATO Conference on Deformation Theory of Algebras and Applications, Il Ciocco, Italy, 1986, pp. 375-401, Kluwer, Dordrecht, 1988.

[7] Fialowski, A.: Deformations of some infinite-dimensional Lie algebras. J. Math. Phys. 31, 1340-1343 (1990).

[8] Fialowski, A., and Fuchs, D.: Singular deformations of Lie algebras. Example: Deformations of the Lie algebra $L_{1}$. In: Topics in Singularity Theory, V.I. Arnold's 60th Anniversary Collection, Transl. Ser. 2, Am. Math. Soc. 180(34), pp.77-92, 1997.

[9] Fialowski, A., and Fuchs, D.: Construction of miniversal deformations of Lie algebras. J. Funct. Anal. 161, 76-110 (1999).

[10] Fialowski, A., and Schlichenmaier, M.: Global deformations of the Witt algebra of Krichever Novikov type. Comm. Contemp. Math. 5, 921-945 (2003).

[11] Fuchs, D.: Cohomology of Infinite-dimensional Lie Algebras, Consultants Bureau, N.Y., London, 1986.

[12] Gerstenhaber, M.: On the deformation of rings and algebras I,II,III Ann. Math. 79, 59-10 (1964), 84, 1-19 (1966), 88, 1-34 (1968).

[13] Gorbatsevich, V.V.,Onishchik, A.L, and Vinberg, E.B : Structure of Lie groups and Lie algebras. In: Onishchik, A.L., and Vinberg, E.B. (eds) Lie Groups and Lie Algebras III. Encyclopaedia of Math. Sciences, Vol. 41, Springer, New-York, Berlin, Heidelberg, Tokyo, 1994.

[14] Hartshorne, R.: Algebraic Geometry, Springer 1977.

[15] Harrison, D.: Commutative algebras and cohomology Trans. Amer. Math. Soc. 104, 191-204 (1962). 
[16] Kac, V.G., Infinite dimensional Lie algebras. Cambridge Univ. Press, Cambridge, 1990.

[17] Kontsevich, M.: Deformation quantization of algebraic varieties. Lett. Math. Phys. 56, 271-294 (2001).

[18] Krichever I.M., and Novikov S.P.: Algebras of Virasoro type, Riemann surfaces and structures of the theory of solitons, Funktional Anal. i. Prilozhen. 21, 46-63 (1987); Virasoro type algebras, Riemann surfaces and strings in Minkowski space. Funktional Anal. i. Prilozhen. 21, 47-61 (1987); Algebras of Virasoro type, energy-momentum tensors and decompositions of operators on Riemann surfaces, Funktional Anal. i. Prilozhen. 23, 46-63 (1989).

[19] Kodaira, K.: Complex manifolds and deformation of complex structures, Springer, New-York, Berlin, Heidelberg, Tokyo, 1986

[20] Lecomte, P.B.A., and Roger, C.: Rigidity of current Lie algebras of complex simple type, J. London Math. Soc.(2) 37, 232-240 (1988).

[21] Nijenhuis, A., Richardson, R.W,: Cohomology and deformations of algebraic structures. Bull. Amer. Math. Soc. 70 (1964), 406-411.

[22] Nijenhuis, A., Richardson, R.W,: Cohomology and deformations in graded Lie algebras. Bull. Amer. Math. Soc. 72 (1966), 1-29.

[23] Palamodov, V.P.: Deformations of complex structures. In: Gindikin, S.G., Khenkin, G.M. (eds) Several complex variables IV. Encyclopaedia of Math. Sciences, Vol. 10, pp. 105-194, Springer, NewYork, Berlin, Heidelberg, Tokyo, 1990

[24] Rauch, G.: Effacement et déformation. Ann. Inst. Fourier, Grenoble 22,1 (1972), 239-269.

[25] Richardson, R.W,: On the rigidity of semi-direct products of Lie algebras. Pac. Jour. of Math. 22 (1967), 339-344.

[26] Ruffing, A., Deck, Th., and Schlichenmaier, M.: String branchings on complex tori and algebraic representations of generalized Krichever-Novikov algebras. Lett. Math. Phys. 26), 23-32 (1992).

[27] Schlichenmaier, M.: Verallgemeinerte Krichever - Novikov Algebren und deren Darstellungen, University of Mannheim, June 1990.

[28] Schlichenmaier, M.: Krichever-Novikov algebras for more than two points. Lett. Math. Phys. 19, 151-165 (1990).

[29] Schlichenmaier, M.: Krichever-Novikov algebras for more than two points: explicit generators. Lett. Math. Phys. 19, 327-336 (1990).

[30] Schlichenmaier, M.: Central extensions and semi-infinite wedge representations of Krichever-Novikov algebras for more than two points, Lett. Math. Phys. 20, 33-46 (1990).

[31] Schlichenmaier, M.: Degenerations of generalized Krichever-Novikov algebras on tori, Jour. Math. Phys. 34, 3809-3824 (1993).

[32] Schlichenmaier, M., Differential operator algebras on compact Riemann surfaces. Generalized Symmetries in Physics (Clausthal 1993, Germany) (H.-D. Doebner, V.K. Dobrev, and A.G. Ushveridze, eds.), World Scientific, 1994, pp. 425-434.

[33] Schlichenmaier, M.: Local cocycles and central extensions for multi-point algebras of Krichever-Novikov type. J. reine angew. Math. 559, 53-94 (2003).

[34] Schlichenmaier, M.: Higher genus affine algebras of Krichever-Novikov type, Moscow Math. J. 3 (4) (2003), 1395-1427, math/0210360

[35] Schlichenmaier, M., and Sheinman, O.K.: The Sugawara construction and Casimir operators for Krichever-Novikov algebras., J. Math. Sci., New York 92, no. 2, 3807-3834 (1998), q-alg/9512016

[36] Schlichenmaier, M., and Sheinman, O.K.: Wess-Zumino-Witten-Novikov theory, KnizhnikZamolodchikov equations, and Krichever-Novikov algebras. Russian Math. Surv. (Uspeki Math. Naukii). 54, 213-250 (1999), math.QA/9812083

[37] Schlichenmaier, M., and Sheinman, O.K.: Knizhnik-Zamolodchikov equations for positive genus and Krichever-Novikov algebras. (to appear in Russian Math. Surv. (Uspeki Math. Naukii)), math.AG/0312040

[38] Tsuchiya, A., Ueno, K., Yamada, Y., Conformal field theory on universal family of stable curves with gauge symmetries. Adv. Stud. Pure Math. 19 (1989), 459-566. 
(Alice Fialowski) Department of Applied Analysis, Eötvös Loránd University, Pázmány PÉTER SÉtÁNy 1, H-1117 BudAPest, Hungary

E-mail address: fialowsk@cs.elte.hu

(Martin Schlichenmaier) Université du Luxembourg, Laboratoire de Mathematiques, Campus Limpertsberg, 162 A, Avenue de la Faiencerie, L-1511 Luxembourg

E-mail address: Martin.Schlichenmaier@uni.lu 